\title{
Die Zerlegung des Ytterbiums in seine Elemente
}

von

\author{
C. Auer v. Welsbach,
}

k. M. k. Akad.

(Mit 3 Tafeln.)

(Vorgelegt in der Sitzung am 19. Dezember 1907.)

\section{Das Trennungsverfahren.}

In meiner im vorigen Jahre erschienenen Arbeit: «Über die Elemente der Yttergruppe, I. Teil « habe ich auf p. 11 erwähnt, daß aus den gereinigten Endmutterlaugen der Hauptreihen als letztes Glied aller Ytterbiumfraktionen ein Präparat gewonnen worden war, dessen Funkenspektrum, verglichen mit dem normalen Yb-Spektrum, auffallende Veränderungen zeigte. So trat beispielsweise die dem Ytterbium so charakteristische 4-Liniengruppe im Grün $(\lambda=5353 \cdot 0,5347 \cdot 4$, $5345 \cdot 9,5335 \cdot 2)$ stark zurück; andere, allerdings meist schwächere Linien fehlten ganz.

Dieses eigentümliche Spektralbild, das vornehmlich bei geringer Lichtstärke des Funkens mit großer Deutlichkeit hervortrat, verlor sich in den folgenden Fraktionen wieder mehr und mehr.

Da nun das Funkenspektrum jener Endfraktion ausschiließlich aus Ytterbiumlinien bestand, so mußte aus den Intensitätsänderungen, die die Linien erfahren hatten, geschlossen werden, daß ein Bestandteil des Ytterbiums sich in den leichter löslichen Fraktionen angereichert habe, daß somit das Ytterbium kein einheitlicher Körper sei. 
In gleichem Sinne sprachen übrigens schon lange vor jenem beweisenden Spektralbefund auch andere Beobachtungen. So war es beispielsweise höchst auffallend, daß der Trennungsprozeß $z$ wischen Erbium-Thulium einerseits und Ytterbium andrerseits sich um so träger gestaltete, je länger er fortgesetzt wurde. Während anfangs schon nach wenigen Reihen farblose, kein Absorptionsspektrum gebende Ytterbiumsalze gewonnen werden konnten, gelang dies später immer schwerer, trotzdem die als rein abgestellten Ytterbiumfraktionen ihrer Menge nach fast gleich geblieben waren. War es nun festgestellt, daß sich beim Fraktionieren der Doppelammonoxalate ein Bestandteil des Ytterbiums - ich will ihn vorerst mit $\mathrm{Yb}$ I bezeichnen - in den leichter löslichen Fraktionen angereichert habe, so mußte naturgemäß bei entsprechend lange fortgesetztem Fraktionieren in den schwerer löslichen Doppelsalzen eine Verminderung dieses Bestandteiles, beziehungsweise eine Vermehrung einer anderen Ytterbiumkonstituente eintreten.

Diese schwerer löslichen Doppelsalze sind aber jene wenig Erbium enthaltenden Fraktionen, die zwischen den farblosen Ytterbium- und den Er-reicheren Fraktionen stehen. Als feste Salze zeigen diese Fraktionen alle neben den Tu-Banden jenes merkwürdige, vom Absorptionsspektrum des reinen Er-Salzes ganz verschiedene Spektrum, das ich in der oben zitierten Abhandlung mit Spektrum Er $\gamma$ bezeichnet habe.

Diese Fraktionen nahm ich nun zunächst in Arbeit. An sie reihte ich später auch alle jene Erbiumfraktionen an, in deren Spektren sich keine Holmiumlinien mehr erkennen ließen.

Das Fraktionieren wurde in ähnlicher Weise wie früher vorgenommen. Da es nach 50 Reihen etwa immer schwieriger wurde, völlig farblose Fraktionen zu erhalten, die Zahl der Fraktionen der Reihe also beträchtlich stieg, so ließ ich zunächst die ganz schwach rosa gefärbten, nur das oben erwähnte Er $\gamma$-Spektrum gebenden Fraktionen abstellen und sie in mehreren Sammelfraktionen vereinigen.

Die Mutterlaugen dieser Reihen wurden eingedampft und gaben kleine Mengen völlig farbloser Fraktionen, die den früher gewonnenen $\mathrm{Yb}$-Fraktionen angegliedert wurden. 
Nach der 75. Reihe ließ ich bereits Salze abstellen, die stärker rosa gefärbt waren als die früher abgestellten und die alle schon neben dem Spektrum Er $\gamma$ in geringem Maße die dem Er-Spektrum charakteristischen Banden zeigten.

Die Mutterlaugen dieser Reihen wurden nicht mehr mit den anderen vereinigt; sie lieferten nach wiederholtem Eindampfen schließlich eine das Speiktrum des Er kaum mehr zeigende Sammelfraktion: Mutterlauge I.

Unter Abstellen der mittleren reinen Erbiumfraktionen, auf die ich mich in dieser Arbeit nicht weiter mehr beziehe, ließ ich die Kristallisationsprozesse allmählich zu Ende gehen. Diese letzteren Reihen lieferten schließlich eine Endmutterlauge II und eine größere Menge schwach rosa gefärbter Salze, die alle das Spektrum Er $\gamma$ gaben.

Die Mutterlauge I gab, mit Salpetersäure gefällt, ein fast weißes Oxalat. Es enthielt hauptsächlich Ytterbium.

Die Mutterlauge II gab ein sehr schwach rosa gefärbtes Oxalat.

In allen diesen Fraktionen war eine kleine Menge zinnähnlicher Elemente enthalten. Ich komme auf diese interessanten Körper, die sich in allen vornehmlich die Elemente der Yttergruppe enthaltenden Mineralien finden, in einer besonderen Abhandlung zurück.

Im Funkenspektrum des reinen, aus dem Oxalate der Mutteriauge II dargestellten Oxydes zeigte sich nun mit.prägnanter Klarheit das Gegenbild des eingangs geschilderten Spektrums. Alle Ytterbiumlinien, die in jenem Spektrum verblaßt waren, traten in diesem mit gesteigertem Glanze auf, so namentlich die 4-Liniengruppe im Grün. Noch glänzender zeigte sich das Spektrum dieser zweiten Ytterbiumkonstituente - sie sei vorerst mit Yb II bezeichnet - in den schwach rosa gefärbten Fraktionen, die sich an die Mutterlauge II anreihten. In den Spektren dieser Präparate ließen sich die Linien, die das eingangs besprochene Spektrum so glänzend erscheinen ließen, kaum mehr wahrnehmen.

Da diese Spektren neben einigen Er-Linien im wesentlichen nur die Linien von $\mathrm{Yb}$ II enthielten, so ließ sich schließen, daß der die Rosafärbung dieser Fraktionen bedin- 
gende Körper entweder kein empfindliches Funkenspektrum besitze oder nur in Spuren zugegen sei. Die erstere Annahme sprach für Er. Das Spektrum dieses Körpers ist im optischen Teile, obzwar es zahlreiche Linien enthält, bekanntlich kein besonders glänzendes. Da nun andrerseits der das Spektrum Er $\gamma$ bedingende Körper sich in den Fraktionen nicht merklich anreichern ließ, so lag es nahe, zu vermuten, daß dies sonderbare Spektrum, das schon so oft zu Täuschungen Veranlassung gab, ein Variationsspektrum des Er sei.

Als ich nun ein Gemenge von zehn Teilen reinsten $\mathrm{Yb}$ Ammonoxalats und einem Teile reinsten Er-Ammonoxalats in möglichst wenig Ammonoxalat enthaltender Lösung kochend löste und dann rasch kristallisieren ließ, bildete sich ein Doppelsalz, das sich weder in seiner Färbung noch in seinem Absorptionsspektrum wesentlich von dem der vorliegenden Fraktionen mehr unterschied.

Der Vollständigkeit halber sei noch erwähnt, daß die Spektren aller ein wenig Er enthaltenden Fraktionen ziemlich deutlich auch die dem Thulium zugeschriebenen Absorptionsbänder zeigten.

Durch die eben geschilderten Versuche war es erwiesen, daß alle schwach rosa gefärbten Fraktionen etwa zehnmal mehr Yb II enthielten als Erbium. Das Spektrum Er. $\gamma$ war von nun ab nur mehr ein bequemer Indikator für den Yb-Gehalt der Fraktionen.

Bei dem hohen Gehalte der schwach rosa gefärbten Fraktionen an Yb II lag es nahe, die Abscheidung dieser Konstituente des Ytterbiums mittels des Verfahrens der überbasisch salpetersauren Salze --- eine zur Gewinnung des $\mathrm{Yb}$ erprobte Methode - zu versuchen.

Nachdem ein mit einem weniger reinen Salze angestellter Vorversuch die Brauchbarkeit dieses Verfahrens zu bestätigen schien, nahm ich diese Fraktionen in der angedeuteten Weise in Arbeit.

$\mathrm{Da}$ ich die richtige Ausführung dieses Verfahrens in meinen früheren Arbeiten bereits geschildert habe, so begnüge ich mich an dieser Stelle, nur kurz über die Resultate zu berichten. Anfänglich ging die Trennung der Bestandteile in 
normaler Weise vor sich; sowie aber der Gehalt an Er unter $1 / 2$ Prozent etwa gesunken war, wurde die Trennung immer schwieriger, so daß es nur durch vielfaches Fraktionieren möglich gewesen wäre, völlig farblose, Er-freie Produkte zu erhalten. Dieses zum Teil negative Ergebnis beweist, daß die Trennung mit Hilfe der Doppelammonoxalate selbst in diesem für die Scheidung durch die überbasisch salpetersauren Salze so günstigen Falle nicht nur die bequemere, sondern auch die weit bessere Methode ist.

Ich ordnete nun die die Ytterbiumelemente enthaltenden Fraktionen zu einer neuen Reihe. Das war etwas schwierig, weil die Präparate aus verschiedenen Trennungsstadien stammten und einen recht verschiedenen Gehalt aufwiesen. Zuerst brachte ich die oben erwähnten, schwach rosa gefärbten Sammelfraktionen in eine Reihe, die aus zehn Fraktionen bestand. Diesen stellte ich jene Fraktionen voran, die ich aus den Mutterlaugen der überbasischen Salze dargestellt hatte. Daran schlossen sich dann noch einige andere stärker erbiumhaltige Präparate der letzten Reihe, so daß diese neue Reihe aus etwa 16 Gliedern bestand. Nun begann der Trennungsprozeß von neuem. Während sich dieser entwickelte, ordnete ich inzwischen die übrigen Ytterbiumfraktionen der Hauptreihe. Einige davon ließen im Spektrum keine Spur von Absorptionsstreifen erkennen, andere aber zeigten solche, wenn auch nur in eben erkennbarem Maße. Ich bezeichne im folgenden die diese Absorptionsstreifen bewirkenden Elemente vorerst kollektiv mit $»$ Erbium $«$. Die meisten Fraktionen enthielten viel überschüssiges Ammonoxalat, und zwar viel mehr, als zur Lösung des Salzes in der Wärme notwendig war.

Dieser Überschuß mußte vorerst entfernt werden, weil er die Trennungsoperation aus heißer Lösung erschwert hätte. $\mathrm{Zu}$ diesem $Z$ wecke stellte ich eine bei etwa $90^{\circ}$ gesättigte Lösung des Salzgemenges dar und ließ diese dann bei $60^{\circ}$ im Thermostaten kristallisieren. Nach etwa 12 Stunden wurde die Lösung von dem ausgeschiedenen Ammonoxalat, das eine an der Schale ziemlich fest haftende Kristallmasse bildete, rasch in eine vorgewärmte Schale abgegossen. Durch mehrmaliges Umkristallisieren bei etwa $30^{\circ} \mathrm{C}$. gelang es leicht, das Ammon- 
oxalat fast rein abzuscheiden. In der von den Kristallen abgegossenen Lauge wurden nun andere Fraktionen gelöst, in gleicher Weise, wie oben geschildert, behandelt und so fortgefahren, bis alles in mehreren Partien in Lösung war. Diese Lösungen hielt ich um so konzentrierter, je unreiner sie waren.

Die Abscheidung der ersten Fraktion erfolgte durchaus bei etwa $50^{\circ} \mathrm{C}$. Sobald der Er-Gehalt unter $1 / 3$ Prozent etwa gesunken war, ließ ich die Laugen bei Zimmertemperatur kristallisieren. Die gleichwertigen Fraktionen wurden vereinigt und zu einer neuen Reihe zusammengestellt.

Die aus dieser Reihe stammenden Mutterlaugen wurden fraktionsweise wiederholt eingeengt. Die schließlich gewonnenen Endmutterlaugen, in denen sich neben seltenen Erden auch verschiedene zum Teil aus dem verbrauchten Wasser stammende Verunreinigungen angehäuft hatten, wurden mit Salpetersäure vorsichtig gefällt. Die aus Erdoxalaten und saurem Ammonoxalat bestehende Fällung wurde abfiltriert, mit kaltem, etwas oxalsäurehaltigem Wasser gewaschen, dann in Wasser verteilt und unter Zusatz von Ammoniak in der Hitze gelöst. Durch Vereinigung der gleichwertigen Fraktionen bildete sich, schließlich eine neue Reihe.

Mit dem fortschreitenden Trennungsprozeß konnten nach und nach die Endglieder der anderen Reihe an diese angeschlossen werden, bis unter Abstellung der schwach rosa gefärbten Anfangsglieder sich alle Fraktionen in einer einzigen Reihe vereinigt fanden.

In dieser Reihe war ungefähr ein halbes Kilogramm Ytterbiumoxyd enthalten.

Es wäre überaus langwierig und möchte nur ermüdend wirken, wollte ich eine detaillierte Schilderung des nun folgenden Trennungsprozesses an dieser Stelle geben. Ich beschränke mich deshalb darauf, nur die wichtigsten Stadien des Prozesses kurz hervorzuheben.

Nach Bildung der zehnten Reihe etwa stellte ich die Kristallaggregate der Anfangsfraktionen $a b$ und wiederholte dies fast bei jeder neuen Reihe. Schließlich lagen 40 Kristallfraktionen und eine größere Menge Endmutterlaugen, als Sammelfraktion gewonnen, vor. Die ersten zehn Fraktionen 
bildeten ihrer Menge nach etwa zwei Drittel der Gesamtmenge. Die ersteren zeigten eine eben noch erkennbare Rosafärbung; die folgenden waren wie alle übrigen rein weiß und gaben, direkt beobachtet, selbst bei langer Schichte kein Absorptionsspektrum mehr. Das Funkenspektrum der ersteren Fraktionen enthielt nur die Linien von Yb II. In den nächstfolgenden ließen sich bereits die intensivsten Linien von $\mathrm{Yb}$ I erkennen. Gegen die Mitte zu traten beide Spektren gleich stark auf. Die Endfraktionen lieferten ein glänzendes Spektrum des zweiten Elementes, in welchem aber noch deutlich merkbar die Linien des ersten Elementes enthalten waren. Selbst das Oxyd der Endmutterlauge gab bei starkem Funken noch die typischen Linien von $\mathrm{Yb}$ II. Wenngleich die Trennung somit trotz aller aufgewendeten Mühe noch immer nicht als völlig gelungen zu betrachten war, so schien es mir doch über jeden Zweifel gestellt zu sein, daß die Reindarstellung der beiden Elemente wenigstens im Bereiche der Möglichkeit gelegen sei.

$\mathrm{Da}$ ich aus verschiedenen Versuchen, die ich mit der Endmutterlauge angestellt habe, den Eindruck gewonnen hatte, als hätten gewisse in der Lauge erkennbare Verunreinigungen störend auf den Gang der Trennung gewirkt, so stellte ich zunächst die Erdoxalate wieder rein dar. Die Lauge wurde mit Salpetersäure bis zur Bildung des vierfach sauren Ammonoxalates etwa versetzt und klären gelassen, der Niederschlag später abgesaugt und gewaschen. Die Fällaugen wurden abgedampft und verglüht. Der feuerfeste Rückstand enthieit neben viel Kieselsäure etwas Tonerde, ein wenig Eisen, eine Spur Uran, $\mathrm{Cu}, \mathrm{K}, \mathrm{Na}$ u. dgl. m. Auch eine kleine Menge der Yb-Elemente war darin nachzuweisen.

Die Erdoxalatfällung wurde verglüht. Sie lieferte etwa $8 g$ Oxyd. Dieses wurde in Salpetersäure gelöst, die wasserklare Lösung verdampft und das Nitrat bis zum Auftreten von Stickoxyden vorsichtig geschmolzen. Die erkaltete Schmelze war trübe. Mit wenig Wasser übergossen und erwärmt, löste sie sich zu einer trüben Flïssigkeit. Nach Zusatz von etwas mehr Wasser schied sich daraus beim Aufkochen weißes, überbasisch salpetersaures Salz aus. Nach einiger Zeit wurde dieser Niederschlag abgesaugt und in Salpetersäure gelöst. 
Schwefelwasserstoff erzeugte in der schwach sauren Lösung nur eine ganz geringe Fällung. Das Filtrat wurde in entsprechender Weise mit Natriumthiosulfat gefällt. Da diese erste Fällung im Funkenspektrum die Yb-Elemente erkennen ließ, wurde sie wiederum in Salzsäure gelöst und neuerdings vorsichtig mit Thiosulfat gefällt. Diese der Menge nach sehr kleine Fällung bestand dem Spektralbefunde nach aus fast reinem Thorsalz.

In der Hauptmenge der Nitratlösung bewirkte Schwefelwasserstoff nur eine kaum merkbare Trübung. Aus der Mutterlauge der Thiosulfatfällung wurden zunächst die Hydrate dargestellt, diese dann in Salpetersäure gelöst und mit der Hauptmenge vereinigt. Die in der Nitratiosung enthaltenen Erden unterwarf ich nun anderen, zur Abscheidung von Yb I geeignet scheinenden Trennungsprozessen. Aber ohne jeden Erfolg. Die kleine Menge Yb II, die dieses Präparat noch enthielt, ließ sich nicht im geringsten Maße verändern.

So nahm ich denn die ursprüngliche Trennungsmethode wieder auf. $\mathrm{Da}$ ich schon früher einmal beobachtet hatte, daß die Trennung um so leichter vor sich ging, je weniger die Lösung übersättigt war, so ließ ich nun die Mutterlauge immer etwa acht Tage über den erst ausgeschiedenen Kristallen stehen. Wiederholte Spektralproben zeigten, daß die Trennung in günstiger Weise verlief. Nach vielmaligem Eindampfen war schließlich eine kleine Menge Mutterlauge gewonnen worden, deren Oxyd, rein dargestellt, im sichtbaren Teile bei gewöhnlichem Funken nur das Spektrum Yb I gab. Die hellsten Linien von Yb II konnten erst bei sehr starkem Funken und da nur mehr andeutungsweise erkannt werden.

$\mathrm{Da}$ ich die Absicht hatte, die Trennungsarbeiten zur Gewinnung größerer Mengen von Yb I später nochmals aufzunehmen, so begnügte ich mich fürs erste mit wenigen Reihen. Die Endmutterlauge der zweiten und dritten Reihe lieferte ein der ersten Mutterlauge annähernd gleiches Produkt.

Um festzustellen, ob sich $z$ wischen $\mathrm{Yb}$ I und $\mathrm{Yb}$ II eine andere seltene Erde oder ein anderes Element einreihe, untersuchte ich Fr. 11, 12 und 13 eingehend. Diese Fraktionen enthielten zirka $100 \mathrm{~g}$ Oxyd. Bei $60 \mathrm{~mm}$ langer Schichte zeigte die 
farblose, nahezu konzentrierte Nitratlösung eine Andeutung eines Absorptionsbandes im Rot. Dies verschwand jedoch bei etwas geringerer Dicke der absorbierenden Schichte wieder gänzlich. Das Oxyd dieser Fraktion war weiß und gab kein Glühspektrum. Das Funkenspektrum war gleich dem des reinen $\mathrm{Yb}$.

Schwefelwasserstoff bewirkte keine Fällung, ebensowenig Kaliumsulfat, womit bewiesen war, daß sich jene eigentümliche Sulfide bildenden Elemente nicht vorfanden. Die reine Nitratlösung wurde schließlich wieder mit saurem Ammonoxalat gefällt, das Oxalat in Ammonoxalat unter Ammoniakzusatz gelöst und kristallisieren gelassen.

Zum Zwecke der Reindarstellung des Elementes $\mathrm{Yb}$ II bildete ich aus den Fraktionen 1 bis 10 der letzten Reihe und den aus der eben besprochenen Rückverarbeitung gewonnenen Produkten eine neue Reihe.

Einzelnen Gliedern dieser Reihe wurde Ammonoxalat entzogen, weil sie mehr davon enthielten, als zur Lösung des Doppelsalzes nötig war.

Das Lösen der Kristallfraktionen wurde stets am Wasserbade bewirkt. Die Mutterlauge der Endfraktion jeder Reihe wurde zurückgestellt, die erste Fraktion der Reihe stets mit Wasser aufgenommen. Alle Fraktionen ließ ich 48 Stunden kristallisieren. Sobald die Anfangsfraktion kein oder wenig Ammonoxalat mehr enthielt, sich also nicht oder nicht reichlich mehr im Wasser löste, wurde sie abgestellt. So erhielt ich nach etwa 30 Reihen zehn an Erden sehr reiche Fraktionen. Die ersten ließen im Absorptionsspektrum sowohl Er als auch Tu erkennen. Die vierte Fraktion war wie alle späteren rein weiß. Sie zeigte kein Er-Absorptionsspektrum mehr. Die fünfte gab ein Oxyd, dessen ziemlich konzentrierte Nitratlösung $\left(1 \mathrm{~cm}^{3} 0.5 \mathrm{~g}\right.$ Oxyd) völlig farblos war. Die Lösung zeigte bei $30 \mathrm{~mm}$ langer Schichte nur ein schwaches Absorptionsband im äußersten Rot. Dieses dem Thulium zugeschriebene Band verlor sich in den späteren Fraktionen wieder.

Das Funkenspektrum dieser Fraktionen ließ selbst bei sehr kräftigem Funken im sichtbaren Teile des Spektrums 
keine der charakteristischen Linien des Yb I mehr erkennen. Es traten nur die dem $\mathrm{Yb}$ II eigentümlichen Linien auf.

Da sonach die Reinheit der gewonnenen Präparate die bei okularer Beobachtung gegebene Empfindlichkeitsgrenze bereits erreicht hatte, so wandte ich mich nunmehr $z u$ der im vorliegenden Falle schärfsten Untersuchungsmethode, zur photographischen Aufnahme des ultravioletten Teiles der Spektren. Die von mir hiebei benützten Apparate und Verfahren finden sich am Schlusse dieser Abhandlung besprochen.

Die glasklar entwickelten Platten zeigten die Funkenlinien mit außerordentlicher Schärfe. Durch direkten Vergleich der Spektrogramme ließ sich ohneweiters feststellen, daß, natürich abgesehen von den beiden Elementen gemeinsamen Linien, sowohl $\mathrm{Yb}$ I wie auch $\mathrm{Yb}$ II noch nicht völlig rein waren.

Ich faßte daher die Wiederaufnahme der Trennungsarbeiten ins Auge. Bei $\mathrm{Yb}$ II schien mir dies auch ziemlich aussichtsvoll zu sein. Denn von diesem Körper hatte ich eine ziemlich beträchtliche Menge gewonnen, die groß genug war, um eine neue Trennungsreihe zu ermöglichen. Bei Yb I aber mußte ich nach reiflicher Erwägung zum Teil der geringen Menge wegen, die mir zur Verfügung stand, auf eine Fortsetzung der Arbeiten verzichten. Nachdem ich die so ermüdende Arbeit längere Zeit hatte ruhen lassen, ordnete ich die an Yb II reichen Fraktionen zu einer neuen Reihe.

Leider waren meine Bemühungen von keinem rechten Erfolge begleitet. Trotz zahlreicher Wiederholungen der Kristallisationsprozesse, trotz mannigfacher Abänderungen des Trennungsverfahrens gelang es nicht, Yb II von Yb I völlig $z u$ scheiden. In den Spektrogrammen traten die intensiven Linien von $\mathrm{Yb} I$ stets wiederum auf. So gab ich denn die weiteren Trennungsversuche auf. Bei Beurteilung der Reinheit der vorliegenden Präparate muß man sich indessen gegenwärtig halten, daß durch die photographische Aufnahme der Spektren die Empfindlichkeit der. Prüfung, man kann fast sagen, beliebig hoch gesteigert werden kann. Hängt es ja doch bei höchst empfindlichen Platten wesentlich nur von der Belichtungszeit $a b$, ob eine Linie auf der Platte sich stark 
abzeichnet oder nicht. Wenn man nun aber berücksichtigt, daß einzelne Linien der beiden neuen Elemente auf die photographische Platte so stark wirken, daß selbst schon bei ziemlich schwachem Funken eine nur eine Sekunde währende Belichtung genügt, die Linie deutlich aufzunehmen, so ist es schließlich leicht begreiflich, daß bei einer Belichtung von 200 und mehr Sekunden die so überaus schwierig zu trennenden Elemente sich nicht als völlig frei voneinander erwiesen.

Ich beabsichtige, die in dieser Arbeit geschilderten Trennungsverfahren mit noch reichlicherem Materiale, als es mir bis jetzt zu Gebote stand, wiederholen zu lassen, teils um anderen Forschern $\mathrm{zu}$ weiteren Messungen genügend reine Proben zur Verfügung stellen zu können, teils um zu erforschen, ob bei weitest getriebener Fraktionierung sich Yb I nicht noch erheblich reiner wird gewinnen lassen. Die Durchführung dieser überaus mühsamen Arbeiten dürfte sechs bis acht Jahre in Anspruch nehmen.

Wie aus der unten folgenden Besprechung der Funkenspektren der beiden neuen Elemente hervorgeht, scheint weder $\mathrm{Yb}$ I noch $\mathrm{Yb}$ II mit unseren heutigen Hilfsmitteln einer weiteren Spaltung fähig zu sein.

Da somit die Zerlegung des Ytterbiums in zwei neue Elemente in experimentell einspruchsloser Weise erfolgt ist, so empfiehit es sich, die Bezeichnung Ytterbium in Zukunft nur mehr als Sammelname zu gebrauchen, den neuen Körpern aber neue Namen zu geben. Ich beantrage für das an das Thulium, beziehungsweise Erbium sich anschließende, in dem vorstehenden Teile dieser Abhandlung mit Yb II bezeichnete Element die Benennung:

Aldebaranium mit dem Zeichen Ad

und für das zweite, in dieser Arbeit mit $\mathrm{Yb}$ I bezeichnete Element, das letzte in der Reihe der seltenen Erden, die Benennung:

Cassiopeïum mit dem Zeichen Cp. 
Was die chemischen Eigenschaften der beiden Elemente betrifft, so kann ich mich kurz fassen. Wer die Chemie des Ytterbiums kennt, kennt auch die der neuen Elemente. Chemisch können sie voneinander durch keine auch noch so sorgfältig ausgeführte Reaktion unterschieden werden. Sie bilden nur ein Oxyd, das in der Glühhitze beständig ist; es ist ein Sesquioxyd. Von diesem leiten sich alle Salze ab. Sie sind farblos, wenn die Säure nicht gefärbt ist.

\section{Die Atomgewichte.}

Auch in ihren Atomgewichten stehen sich die beiden Elemente überaus nahe.

Die nach der Bunsen'schen Methode ausgeführten Bestimmungen ergaben für Aldebaranium:

$$
\mathrm{O}=16 .
$$

Analyse I: $\quad$ Oxyd $=0.4181$, Sulfat $=0.6730 \ldots \ldots 172 \cdot 98$ Analyse II: Oxyd $=0.5984$, Sulfat $=0.9634 \ldots \ldots \ldots 172.88$ Analyse III: Oxyd $=0.6173$, Sulfat $=0.9939 \ldots \ldots .172 \cdot 85$ als Mittel $\mathrm{Ad}=\mathbf{1 7 2 \cdot 9 0}$

für Cassiopeïum:

Analyse I: $\quad$ oxyd $=0.3716$, Sulfat $=0.5967 \ldots \ldots 174.25$ Analyse II: Oxyd $=0 \cdot 3086$, Sulfat $=0 \cdot 4956 \ldots \ldots 174 \cdot 19$ Analyse III: Oxyd $=0 \cdot 4026$, Sulfat $=0.6465 \ldots \ldots 174.24$ als Mittel $\mathrm{Cp}=\mathbf{1 7 4} \cdot \mathbf{2 3}$

Zu diesen Bestimmungen habe ich folgendes zu bemerken:

Das Oxyd der Analyse I des Ad entstammte einem Oxalate, das durch mehrmalige Fällung aus stark salpetersaurer Lösung erhalten worden war.

Die Oxyde der Analysen II und III des Ad sowie die Oxyde der Analysen des $\mathrm{Cp}$ wurden aus einer $\mathrm{CO}_{2}$-freien, viel Ammonnitrat enthaltenden Nitratlösung mit $\mathrm{CO}_{2}$-freiem Ammoniak wiederholt gefällt.

Die Genauigkeit der Atomgewichtsbestimmungen dieser Elemente wird, abgesehen von der Höhe des Atomgewichtes selbst, auch dadurch im ungünstigen Sinne beeinflußt, daß die 
Glühbeständigkeit der Sulfate dieser Elemente gegenüber den Sulfaten anderer seltenen Erden etwas geringer ist.

\section{Die Funkenspektren.}

Zur Erzeugung des leuchtenden Dampfes bediente ich mich ausschließlich meiner neuen Funkenapparate, die sich bekanntlich auf die Anwendung des Öffnungsfunkens stützen und die es bei richtiger Anordnung und unter Verwendung rotierender Platinpole leicht ermöglichen, die Spektren der meisten Elemente fast rein und frei von den Linien der Polsubstanz sowie völlig frei von den Luftlinien zu erhalten.

Ihrer Art nach stehen die Spektren der Öffnungsfunken in mancher Beziehung gewissermaßen zwischen den Bogenspektren und den eigentlichen Funkenspektren. Sie nähern sich in hohem Maße aber sonderbarerweise, wenigstens bei der von mir gewählten Anordnung, einmal mehr den ersteren, dann wieder den letzteren.

So ist das Funkenspektrum des Platins z. B. fast identisch mit dem Bogenspektrum, während die Öffnungsfunkenspektren der seltenen Erden beispielsweise ganz mit den. Funkenspektren, wie sie Exner und Haschek erzeugt und gemessen haben, übereinstimmen.

Die Linien des Bogenspektrums des Platins koinzidierten völlig mit den Linien des Öffnungsfunkenspektrums des Platins. In keinem Falle konnte auch nur die kleinste Verschiebung ermittelt werden.

Die Funkenspektren der beiden neuen Elemente sind relativ linienarm, doch zäblen sie $z u$ den glänzendsten, die man kennt. Dies gilt namentlich für das Cassiopeüum. Die wenigen Linien, die dieser Körper im optischen Teile des Spektrums hat, erinnern etwas an das prachtvolle Spektrum des Baryums. Neben diesen Linien ist das Cp-Spektrum noch durch zwei kannelierte, hellstrahlende Banden im Grün und Blau, die etwas an das Cy-Spektrum erinnern, gekennzeichnet. Während die im Grün liegende Bande aus zahlreichen feinen Linien besteht, ist jene im Blau nebliger Art. Bei der Dispersion, die meine Apparate besitzen, gelang die Auflösung in Linien nicht. Die Lichtmaxima liegen nach dem brechbareren 
Teile des Spektrums zu, der Struktur der Cy-Banden also entgegengesetzt.

Das Spektrum des Ad ist sowohl im optischen als auch im ultravioletten Teile weit linienreicher als das des Cp. Ihm fallen die meisten Linien des $\mathrm{Yb} z \mathrm{u}$. Zur Erzeugung des Spektrums diente bei allen für die Reproduktion oder Messung gemachten photographischen Aufnahmen ein Rowland'sches Konkavgitter älterer Art. Es besitzt 14473 Furchen pro engl. Zoll und einen Krümmungsradius von zirka $2 \mathrm{~m}$. Die Länge der geteilten Fläche beträgt zirka $54 \mathrm{~mm}$. Ich gab diesem Gitter, mit dem ich auch alle vergleichenden Aufnahmen der letzten Jahre gemacht habe, den Vorzug vor anderen, weil es neben ausgezeichneter Definition, die für meine Spektralarbeiten in hohem Maße wertvolle Eigenschaft besitzt, das gebeugte Licht nicht gleichmäßig zu verteilen, sondern es in gewissen, für die Aufnahme günstig gelegenen Spektralbezirken zu konzentrieren. ${ }^{1}$ Infolge dieses Umstandes gelang es mir oft, Spektralaufnahmen von brillanter Schärfe für die ganze Länge des Spektrums unter Verbrauch nur weniger Milligramme der Versuchssubstanz zu machen.

Läßt man in der Richtung der optischen Achse etwa einen Sonnenstrahl auf dieses Gitter fallen und reflektiert das gebeugte Licht gegen eine Wand, so erweist sich das Spektrum der ersten Ordnung auf der einen Seite als überaus lichtstark, während das der anderen Seite ganz verblaßt ist, und dies gilt in gleicher Weise auch für die Spektren der nächst höheren Ordnungen. Sonderbarerweise bricht nun das Spektrum der 1. Ordnung bei ungefähr $\lambda 3300$ plötzlich $a b$, findet sich aber in der 2. Ordnung derselben Seite mit so großer Intensität wieder, daß die photographische Aufnahme für die 1. und 2. Ordnung gleichzeitig mit fast gleichbleibendem Effekt gemacht werden kann. Infolge dieses Umstandes habe ich für den Spektralbezirk von 3300 nach den kürzeren Wellenlängen hin stets in der 2. Ordnung aufgenommen.

Die hohe Empfindlichkeit unserer gewöhnlichen Trockenplatten für kurzwelliges Licht bringt es mit sich, daß die Zone,

1 Vergl. H. Kays er, Handbuch der Spektroskopie. I. Bd., p. 433. 
innerhalb welcher Linien der 1. und 2. Ordnung gemeinsam zur Aufnahme gelangen, eine ziemlich große, etwa $800 \mathrm{~A}$. E. umfassende ist.

Da es nun bei vielen Untersuchungen, wie $z$. B. bei Prüfung höchst kostbarer Substanzen, von großem Werte ist, einen mehrere tausend A. E. umfassenden Spektralbezirk in einer einzigen, zur Vergleichung oder Messung direkt geeigneten Aufnahme unter möglichst reichlicher Ausnützung des Funkenlichtes zu erhalten, habe ich mich zur Sammlung des Lichtes statt der üblichen Quarzlinsen innen versilberter Hohlspiegel bedient.

Alle Wellen von $235 \mu \mu$ abwärts werden, weil Silber für solche Strahlen bekanntlich durchlässig ist, vom Spiegel nicht in erheblichem Maße reflektiert, wodurch $\lambda 4600$ der 1 . Ordnung sicher frei von Linien der 2. Ordnung erhalten werden konnte. Der durch eine Aufnahme auf gewöhnlichen Bromplatten festgehaltene, zur Messung direkt taugliche Teil des Spektrums reichte sonach von $\lambda 4600$ bis 2500 . Die Anwendung von Hohlspiegeln empfiehlt sich der großen Lichtstärke halber, welche die Spektren hiedurch erhalten, besonders auch dann, wenn die auf die gewöhnliche Platte nicht wirksamen Teile des sichtbaren Spektrums aufgenommen werden sollen. Das aber ist, abgesehen von den Messungen der Wellenlängen, für viele vergleichende chemische Untersuchungen von großer Bedeutung. Denn gerade in diesem Teile des Spektrums kündigen sich die durch beginnende Mischungsänderungen hervorgerufenen Intensitätsunterschiede der Linien zweier Spektren am frühesten an, lange bevor sie im ultravioletten Teile erkannt werden können.

Die dieser Abhandlung beigegebenen Tafeln sprechen für die Richtigkeit des eben Gesagten in der deutlichsten Weise. Ich werde diese Verhältnisse weiter unten nochmals zur Sprache bringen.

Gitter, Spalt sowie das Gehäuse für die biegsame, aus federndem Material erzeugte Kassette sind auf einem starken, aus völlig trockenem Mahagoniholz gebauten, gesplindeten Rahmen fix zueinander aufgestellt. Der ganze Apparat ist von einem geräumigen, gleichfalls aus Mahagoniholz gemachten 
Gehäuse, das innen mit schwarzem glanzlosen Samt ausgeschlagen ist, umschlossen. Der Rahmen ruht, auf Kautschukballen frei liegend, auf einem aus Winkelblech erzeugten Stativ. Der ganze Apparat ist nach allen Seiten hin einstellbar und nicht schwieriger $z u$ transportieren als ein anderer großer Spektralapparat. Ich komme auf die ganze Einrichtung, die manches Neue bietet, in meiner demnächst zu veröffentlichenden Arbeit: »Spektroskopische Methoden der analytischen Chemie nochmals zurück.

Fast alle Aufnahmen wurden auf hochempfindlichen, etwa $0.8 \mathrm{~mm}$ dicken, somit leicht biegsamen Trockenplatten von feinstem Korn gemacht. Da sich Glasplatten von solcher Dünne mittels eines geeigneten Diamanten bei nur einiger Übung ohne Splittern des Randes haarscharf schneiden lassen, so gelingt es leicht, zwei oder mehrere auf derartigen Platten aufgenommene Spektren sehr nahe aneinander zu rücken, wodurch man sie, vorausgesetzt, daß deren Aufnahme unter den ganz gleichen Versuchsbedingungen gemacht worden ist, bequem direkt miteinander vergleichen kann.

Die fixe Lage zwischen Gitter, Spalt und Platte bedingte es, daß nur eine gewisse Region der Spektren normal war.

Ich legte diesem Umstande kein großes Gewicht bei, weil es ursprünglich nicht in meiner Absicht gelegen war, ganze Spektren zwecks Ermittlung genauer Wellenlängen durchzumessen, ich vielmehr in erster Linie darauf Bedacht nahm, die Spektren in direkt vergleichbarer Form zu gewinnen. Für alle jene Fälle, bei denen sich Messungen von Linien nicht umgehen ließen oder wo es sich darum handelte, den Ort einer Linie genau zu ermitteln, wollte ich ohne große Mühe und ohne umfangreiche Hilfsmittel innerhalb weniger Minuten den gewünschten Aufschluß erhalten. Deshalb habe ich mich von den üblichen bewährten Komparatormethoden emanzipiert und maß die Spektren nach photographierten, der Längenausdehnung der Spektrogramme fast genau entsprechenden Skalen aus. Allein die geringe Schärfe, die die von gedruckten Skalen abgenommenen Teilstriche anfangs hatten, setzte nun freilich die Genauigkeit der Messung oft über Erwarten herab und beanspruchte zudem das Auge in sehr empfindlicher Weise. 
Durch einige Kunstgriffe gelang es mir indes spüter, diesem Übelstand abzuhelfen und die Skalen so scharf zu photographieren, daß sie ohne besondere Anstrengung des Auges eine beträchtliche Vergrößerung erlaubten und sich somit zu ziemlich genauen Messungen eigneten, namentlich für die Aufnahmen im Spektrum 2. Ordnung, bei denen die A. E. etwa $0.27 \mathrm{~mm}$ des Photogrammes entsprach. Als Standardlinien benützte ich die Platinlinien. Die Wellenlängen dieser entnahm ich den Tabellen H. Kayser's über das Bogenspektrum des Platins. Ich rundete diese Zahlen, welche die Wellenlängen bekanntlich bis auf Tausendstel der A. E. angeben, für meine Zwecke auf zwei Dezimalen ab.

Die Differenzen, die zwischen den Wellenlängen von Kayser (Bogenspektrum) und jenen von Exner und Haschek (Funkenspektrum) bestehen, bedingen zumeist die Abweichungen, die zwischen meinen Messungen und denen der letztgenannten Forscher sich ergaben.

Da im optischen Teile die Anzahl der Platinlinien zu gering ist, um brauchbare Messungen zu ermöglichen, benützte ich zur Messung die Linien des Platinspektrums der 2. Ordnung, das ich zwischen den beiden anderen Spektren, stark verkürzt und dadurch leicht kenntlich gemacht, aufnahm. Die im Spektrum der Versuchssubstanz enthaltenen Platinlinien der 1. Ordnung gestatteten dann eine Kontrolle einer eventuellen Verschiebung.

Ein Abweichen der Linien infolge der Höhenlage des Aufnahmeortes ( $800 \mathrm{~m}$ Seehöhe) konnte ich nicht konstatieren.

Die Spaltbreite betrug bei allen Aufnahmen zirka 0.01 mm.

Als Stromquelle diente eine für Lichtbetrieb gebaute Akkumulatorenbatterie von 50 Volt Klemmenspannung. Bei allen in dieser Abhandlung besprochenen Aufnahmen war in den Strom$\mathrm{kreis}$ ein konstanter Widerstand von $25 \mathrm{Ohm}$ eingeschaltet. Die zur Erzeugung der Funken verfügbare Intensität war demnach eine ziemlich geringe, so daß die aus $1.4 \mathrm{~mm}$ dicken Platindrähten gebildeten positiven Pole stets nur an ihren Enden in schwaches Glühen kamen.

Als Sensibilisator für die Aufnahmen im sichtbaren Teile habe ich Äthylrot verwendet. Da über die sensibilisierende. 
Wirkung dieses Farbstoffes verschiedene Publikationen vorliegen, so kann ich sie als bekannt voraussetzen. Ich habe zumeist ihrer hohen Empfindlichkeit wegen mit feuchten Platten gearbeitet, und zwar exponierte ich sie immer unmittelbar nach der Sensibilisierung, weil bei längerem Liegen, vermutlich durch den $\mathrm{CO}_{2}$-Gehalt der Luft, die Empfindlichkeit alsbald beträchtlich sank. Anhaftende Wassertröpfchen strich ich mit einem breiten, feinen Pinsel ab. Daß die Gradation der sensibilisierten Platten manches zu wünschen übrig ließ, braucht wohl kaum besonders hervorgehoben zu werden.

Teils um die Intensität der Linien besser schätzen, teils um etwa vorhandene Verunreinigungen sicherer erkennen $\mathrm{zu}$ können, habe ich unter möglichst gleichen Versuchsbedingungen, und zwar für sämtliche Bereiche des Spektrums, stets mehrere Aufnahmen bei verschieden langer Expositionszeit gemacht. So für den Bereich von $\lambda 4600$ bis 2500 solche mit Belichtungszeiten von 1, 10,100, 180 und 200 Sekunden, bei $\mathrm{Cp}$ um jede Spur etwa vorhandener Verunreinigungen zu finden, auch eine mit 500 Sekunden. Im sichtbaren Teile begnügte ich mich mit drei Aufnahmen von 60, 200 und 600 Sekunden Belichtungszeit. Die Schätzung der Intensität der Linien in diesem Teile habe ich mitunter auch nach okularer Beobachtung gemacht, weil die sensibilisierten Platten gegenuiber den gewöhnlichen ganz andere, weit unregelmäßiger. verlaufende Empfindlichkeitsgrenzen besitzen. Natürlich entbehren diese ebenso wie die anderen Zahlen jeder wissenschaftlichen Grundlage.

In den folgenden Tabellen habe ich die mit Hilfe der obgenannten Platten erzielten Messungsergebnisse, soweit sie sich auf die intensiveren Linien beziehen, zusammengestellt.

Im Bereiche von 4600 bis 2500 finden sich alle starken Linien verzeichnet, die auf den Platten, deren Belichtungszeit 100 Sekunden betrug, zu sehen waren, ausgenommen diejenigen, die von der Substanz der Pole stammten, wie die Pt-Linien und einige Linien des $\mathrm{Pd}$, Ir und $\mathrm{Cu}$.

Ich habe speziell diese Belichtungszeit gewählt, weil diese Photogramme in Bezug auf die Intensität der Linien den von Exner und Haschek aufgenommenen sehr ähnlich waren 
und somit die von den genannten Forschern gemachten Angaben mit meinen verglichen und ihnen gegenübergestellt werden konnten. Der Vollständigkeit halber habe ich auch jene Wellenlängen aufgenommen, die Exner und Haschek für das Bogenspektrum des Ytterbiums angeben. Die für die chemische Forschung so überaus wichtigen Spektralarbeiten Exner's und Haschek's setze ich im übrigen als bekannt voraus.

Aus den Intensitätsverhältnissen der Linien für den Bereich von 4600 bis 2500 ersieht man, daß die Öffnungsfunkenspektren der in Rede stehenden seltenen Erden den durch hochgespannte Ströme erzeugten Funkenspektren sehr ähnlich sind.

Für alle im Bereiche der 1. Ordnung, d. i. von 4600 bis 3300 , gemachten Messungen habe ich die Exner'schen Zahlen zumeist unverändert angegeben, sofern die von mir ermittelten gegenüber jenen keine größere Differenz aufwiesen als $0.05 \mathrm{~A}$. E. Für die im Bereiche der 2. Ordnung liegenden Messungen habe ich diese zulässige Differenz auf etwa $0.03 \mathrm{~A}$. E. erniedrigt. In diesem Bereiche finden sich demzufolge ziemlich viele Zahlen, die von denen Exner's abweichen. Diese Verschiedenheiten lassen sich aber in den meisten Fällen auf die bereits oben erwähnten, zwischen den Zahlen Kayser's und Exner's bestehenden Differenzen zurückführen.

Zur Ergänzung dieser Tabellen habe ich der vorliegenden Arbeit auch eine bildliche Darstellung der Spektren angefügt. Obzwar die Schwierigkeiten, die einer guten Reproduktion von Spektralphotogrammen im Wege stehen, bekanntlich recht erheblich sind, schienen sie mir dennoch in dem vorliegenden Falle den Vorteilen gegenüber, welche die naturgetreue Abbildung bietet, nicht in Betracht kommen zu sollen. Denn erstens können die Funkenspektren der neuen Elemente sowohl in ihrer Beziehung zueinander als auch zu Ytterbium - und das ist wohl das wichtigste in dieser Arbeit - nur an der Hand guter Bilder kurz und leicht verständlich besprochen werden; zweitens handelt es sich hier um überaus seltene, sehr schwierig darzustellende Elemente, die selbst in den kleinen, zur Funkenerzeugung nötigen Mengen nicht oder 
höchst schwer $\mathrm{zu}$ beschaffen sein werden, deren Spektren aber - und dies gilt besonders für $\mathrm{Cp}$ - durchaus nicht durch die Lage der Linien allein, sondern gerade durch deren Charakter und Beschaffenheit gekennzeichnet werden.

Auf Grund guter Reproduktionen kann man sich zudem mit Hilfe der Wellenlängentafeln leicht für jedes Instrument direkt vergleichbare Aufnahmen machen, mit deren Hilfe ein zu. untersuchendes Spektrum leicht und schnell geprüft werden kann. Das alles aber ist für die chemische Forschung in vielen Fällen von Wichtigkeit. Freilich gilt hiebei als Voraussetzung, daß zur Erzeugung des leuchtenden, das Spektrum gebenden Dampfes stets dieselben Vorrichtungen unter möglichst gleichen Versuchsbedingungen angewendet werden. Sämtliche Bilder sind direkt auf photographischem Wege erhalten worden. Sie umfassen die Region von 6000 bis 2600 .

Zur Entwicklung der Platten benütze ich ausschließlich Hydrochinon. Von dem Bestreben geleitet, die Halbtöne sowie auch die schwächeren Linien voll zur Geltung zu bringen, vermied ich, gemäß den Vorschriften der photographischen Technik, alles, was die Kontraste hätte beträchtlich steigern können. Die Bilder haben dadurch freilich manches Bestechende eingebüßt, an instruktiver Deutlichkeit aber erheblich gewonnen.

Die Linien der Spektren sind ohne jede Retouche und ohne jede Korrektur geblieben, so daß sie einen möglichst getreuen Ausdruck der Spektralerscheinung darbieten, soweit dies eben mit Hilfe der Photographie möglich ist.

Zur Funkenerzeugung verwendete ich ausschließlich reine wässerige Nitratlösungen von ungefähr $10 \%$ Oxydgehalt.

Die über dem Spektrum angebrachte Skala soll lediglich zur leichteren Orientierung dienen; die Wellenlängen selbst müssen daher stets aus der Tabelle entnommen werden.

Die Tafeln von $\lambda .6000$ bis 3300 (1. Ordnung) sind Vergrößerungen im Verhältnisse von $1: 2$, jene von 3300 bis 2600 (2. Ordnung) sind in natürlicher Größe wiedergegeben.

$\mathrm{Zu}$ diesen Aufnahmen verwendete ich dieselben Präparate wie zu den für die Messung bestimmten.

Das erste Spektrum ist das des Ytterbiums (Mittelfraktion). Belichtungszeit für alle Platten war 200 Sekunden. 
Das zweite Spektrum ist Ad, das dritte Cp. Belichtungszeit für alle Platten 180 Sekunden.

Das vierte Spektrum, der Breite nach nur halb so groß gehalten als die anderen, ist das Spektrum des Platins. Belichtungszeit 100 Sekunden.

Die Pole wurden, um die Platinlinien etwas zurückzuhalten, während jeder Aufnahme wiederholt mit Lösung versorgt.

Bei allgemeiner Betrachtung der Bilder fällt zunächst die große Verschiedenheit auf, die die Spektren von Ad und $\mathrm{Cp}$ im optischen Teile, dem Aufnahmsgebiete der sensibilisierten Platten, zeigen. Hier teilt sich das Spektrum des $\mathrm{Yb}$, von der Intensität der Linien natürlich abgesehen, geradewegs in die beiden anderen. Nicht ganz so im violetten und ultravioletten Teile, dem eigentlichen Bereiche unserer hochempfindlichen Bromplatten. Wenngleich sich das Ytterbiumspektrum auch in diesen Regionen ziemlich deutlich als die Summe der beiden anderen Spektren zu erkennen gibt, so sind diese selbst unter sich doch viel äbnlicher. Denn fast jede starke Linie im Spektrum des einen Elementes hat ihre wenn auch meist schwache Gegenlinie in dem Spektrum des anderen. Ich habe diese eigentümlichen, für die Anwendung der Spektralanalyse bei analytisch-chemischen Arbeiten wichtigen Erscheinungen bereits oben berührt.

In dem gegebenen Falle könnte man freilich unter anderem einwenden, daß, weil die Belichtungszeit für sämtliche Platten die gleiche war, die violetten und ultravioletten Strahlen infolge der höheren Lichtempfindlichkeit der Platten eben stärker wirkten als jene im sichtbaren Teile gelegenen. Das mag für die gelben Strahlen richtig sein, für die blaugrünen aber gilt dies nicht. Die eigentlichen Ursachen liegen weit verborgener.

Noch deutlicher treten die betonten Unterschiede bei Betrachtung der Platinlinien hervor. Da zur Funkenerzeugung stets nur Platinpole verwendet wurden, so mußten sich alle auf die Pole gebrachten Substanzen während der Funkenbildung in gewissem Grade mit Platin verunreinigen oder, besser gesagt, bestand die eigentlich funkengebende Substanz aus einer Legierung von Erdmetall und Platin, wie sie sich, nebenbei bemerkt, tatsächlich an den peripherẹn Teilen des 
Poles chemisch nachweisen läßt. Man vergleiche nun das Verhalten der Linien $\lambda 5301 \cdot 18$ im Blaugrün mit jenem der Linie $\lambda .2998 .09 \mathrm{im}$ Ultraviolett. Während die eine trotz ihrer außerordentlichen Intensität im Spektrum der Erden kaum erkennbar ist, ist die andere bei viel geringerer Stärke in allen Spektren scharf und deutlich sichtbar. Um diese merkwürdigen Unterschiede noch markanter zu machen, habe ich die Spektralzone von 4700 bis 4530 doppelt abgebildet, und zwar nach der Aufnahme auf der sensibilisierten Platte, wie auch nach jener auf der gewöhnlichen Bromplatte. Bezüglich der letzteren muß ich bemerken, daß sie absichtlich unter Ausschluß eines Strahlenfilters gemacht worden ist, weshalb sich darin einige schwache, jedoch nicht weiter störende Linien 2 . Ordnung finden. Man beachte nun die Platinlinie 4552.59 in den beiden Teilen.

Im Platinspektrum der sensibilisierten Platte ist diese Linie höçst intensiv, im Erdspektrum dagegen kaum sichtbar; während sie im Pt-Spektrum der Bromplatte wenig stark ist, tritt sie im Erdspektrum scharf und deutlich hervor.

Vergleicht man nun die Photogramme der sensibilisierten Platten okular mit dem Spektrum selbst, so ergibt sich im großen und ganzen eine auffallende Übereinstimmung, eine Erscheinung, die übrigens eine sehr einfache Erklärung zuläßt. Was für die besprochenen Linien gilt, hat auch für alle anderen in Frage kommenden Linien und, soweit ich es heute überblicken kann, für alle Spektren Geltung. Die Empfindlichkeit der Spektralreaktion ist im violetten und ultravioletten Teile des Spektrums, dem Wirkungsbereiche der gewöhnlichen Trockenplatte, weit größer als im sichtbaren Teil und nimmt in diesem gegen das weniger brechbare Ende stetig ab. Deshalb müssen in allen Fällen, wo es sich darum handelt, beginnende Mischungsänderungen festzustellen, die Spektralprüfungen im sichtbaren Teile und zwar okular oder mit möglichst hoch sensibilisierten, nassen Platten gemacht werden. Ich habe diese Verhältnisse in aller Kürze an dieser Stelle zur Sprache gebracht, teils weil sie mir zur richtigen Beurteilung der Tafeln wichtig schienen, teils weil ich glaube, daß sie, bei chemischen Arbeiten bisher außer Acht gelassen, je eher je besser zur allgemeinen Beachtung gelangen sollen. 
Sie erschöpfend zu behandeln, muß ich mir allerdings für eine spätere Arbeit vorbehalten, um so mehr, als dies ohne eingehende Erörterung über die Art der photochemischen Prozesse, die sich in der Platte selbst abspielen und die noch mancher Aufklärung bedürfen, nicht möglich wäre.

In allen Spektren finden sich mehrere Ca-Linien, so insbesondere $\lambda 3968 \cdot 16$ und $3933 \cdot 80$. Die Intensität der ersten Linie wird in den Wellenlängentabellen von Exner und Haschek mit 500, die der letzteren mit 1000 angeführt. Kleine Spuren von Calcium waren übrigens in allen Präparaten nachweisbar. Es gelang durch die bekannten chemisch-analytischen Verfahren nicht, sie völlig den Erden zu entziehen. Andere Methoden, die zur exakten Scheidung geführt hätten, ließen sich mit Rücksicht auf die geringe Menge der vorliegenden Proben nicht anwenden.

Das gleichmäßige Auftreten des Calciums in diesen Fraktionen, die, wie erinnerlich, durch fraktionierte Kristallisation aus ammoniakalischen Ammonoxalatlösungen gewonnen worden waren, war höchst befremdend; ich unterließ es deshalb nicht, das $\mathrm{Ca}$ dieser Präparate näher zu prüfen. Durch vorsichtiges Fällen der gut ausgekochten, reichlich Ammonsalze enthaltenden Erdmetallösung mit $\mathrm{CO}_{2}$-freiem Ammoniak gelang es mir, eine winzige Menge $\mathrm{Ca}$ rein zu extrahieren. Das Funkenspektrum, das diese Probe gab, war identisch mit dem normalen Spektrum des Calciums; keine einzige Linie ließ irgendwelche Intensitätsunterschiede erkennen.

Im ultravioletten Teile des Ad-Spektrums sind Linien vorhanden, die im Yb-Spektrum nicht oder fast nicht zu sehen sind; es sind dies Linien der Thuliumelemente.

Schließlich habe ich noch anzuführen, daß sich in der oberwähnten Aufnahme des Cp-Spektrums, deren Belichtungszeit 500 Sekunden betrug, auch einige ganz schwache FeLinien und eine sehr schwache Mg-Linie erkennbar waren. Linien anderer Körper fanden sich darin nicht.

Mehrere Linien finden sich, namentlich im Ultraviolett, in allen drei Spektren mit ähnlicher Intensität; ich habe sie in den Tabellen mit dem Vermerk »g. L.«, d. h. gemeinsame Linien, besonders hervorgehoben. 
Es ist nicht wahrscheinlich, daß dies nur scheinbare Koinzidenzen sind. Denn diese sonderbare Erscheinung tritt bei allen chemisch einander sehr nahe stehenden Elementen wieder auf. So z. B. auch bei Neodym und Praseodym.

Schließlich habe ich noch einer dritten Gruppe von Linien $\mathrm{zu}$ gedenken, die in den Spektren der Mittelfraktionen am stärksten sind; namentlich auf den sensibilisierten Platten bei langer Belichtungszeit (600 Sekunden) treten sie deutlich hervor.

$\mathrm{Zu}$ diesen zählen beispielsweise die Linien 5104.60, $5067 \cdot 40,5009 \cdot 72$, die in den Spektren von Ad und Cp keine entsprechend markanten Gegenlinien aufweisen. Alle diese Linien sprechen dafür, daß zwischen $A d$ und $C p$ noch eir drittes Yb-Element, wenn auch nicht in besonders reichlichem Maße, sich finde. Diesen Körper in halbwegs reinem Zustande $\mathrm{zu}$ gewinnen, ist leider aussichtslos. Denn zu seiner Darstellung würden die Trennungsverfahren, vorausgesetzt, daß die. Trennungstendenz stetig die gleiche bliebe, was aber durchaus nicht sicher ist, viele Jahrzehnte währen müssen.

Zur Abwehr gewisser Prioritätsansprüche, die man jüngst geltend $\mathrm{zu}$ machen versucht hat, sei mir noch eine kurze persönliche Bemerkung gestattet. Die Zerlegung des Ytterbiums in zwei neue Elemente habe ich anfangs 1905 festgestellt.

Im März desselben Jahres berichtete ich über diese Entdeckung an die kaiserl. Akademie der Wissenschaften in Wien. Dieser zwar kurz, aber ganz bestimmt gefaßte Bericht ist im akademischen Anzeiger Nr. X, Jahrgang 1905, erschienen. Es heißt darin unter anderem wörtlich:

»Es (Yb) besteht hauptsächlich aus zwei Elementen. Die Funkenspektren der neuen Elemente sind Teile des Ytterbiumspektrums und kann dieses als Summe der beiden neuen Spektren gelten. Einzelne Linien scheinen beiden Elementen gemeinsam zu sein.

Dieser Bericht ging später in zahlreiche Fachschriften über und gelangte so zur allgemeinen Kenntnis. 
Wellenlängentafeln.

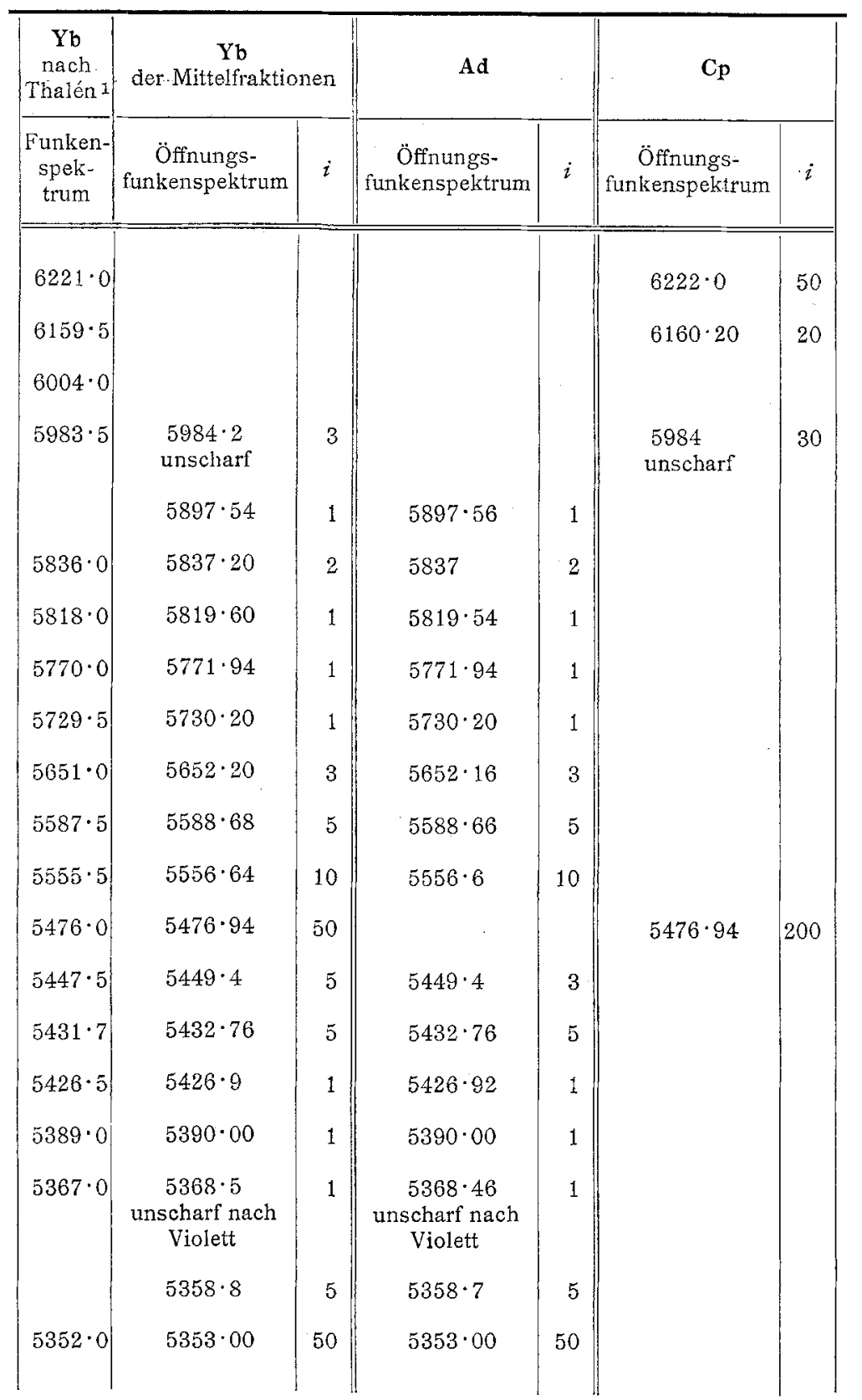

1 Öfvers. K. Vetensk. Akad. Förhandl. 1881. 


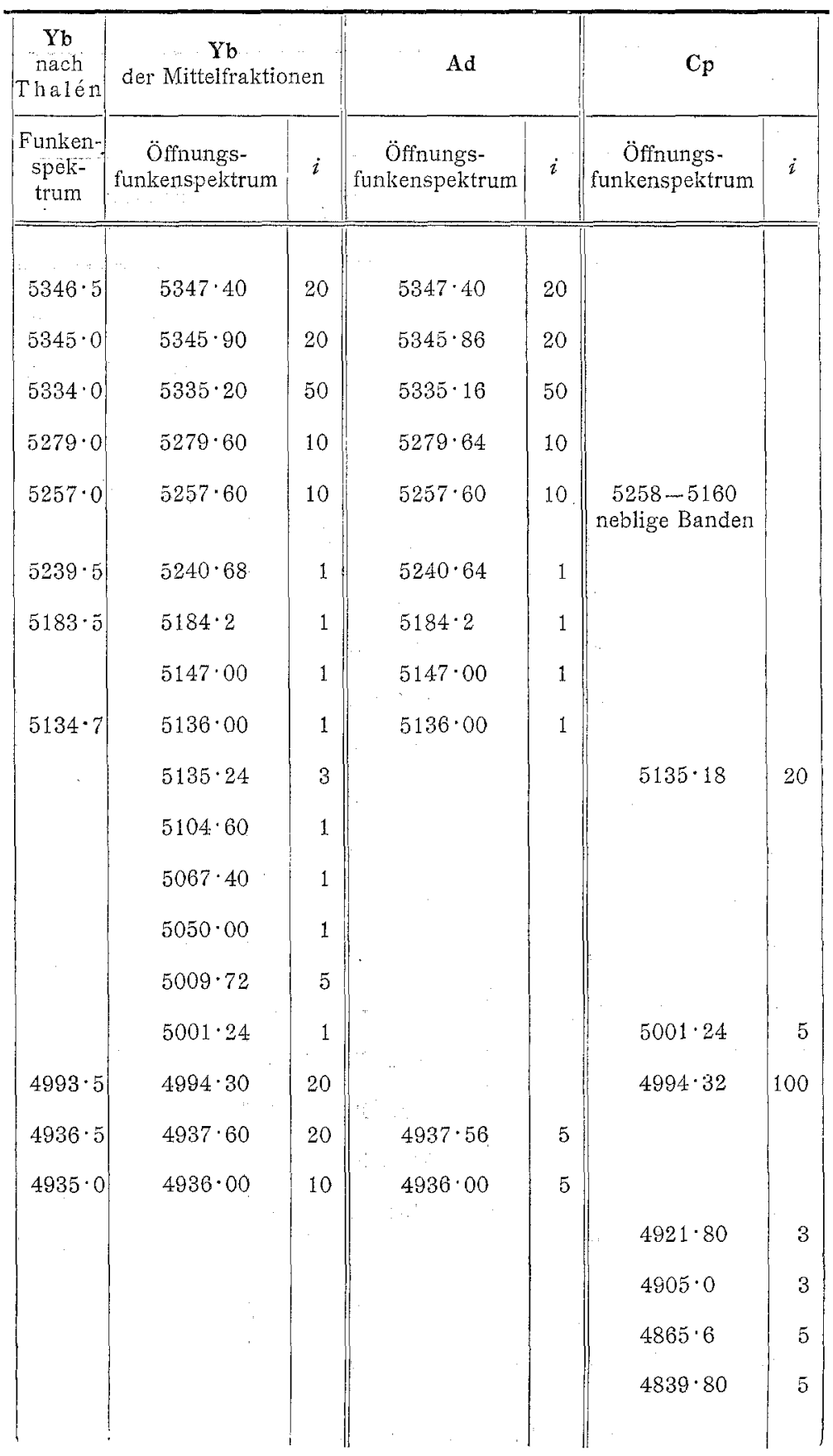


Zerlegung des Ytterbiums.

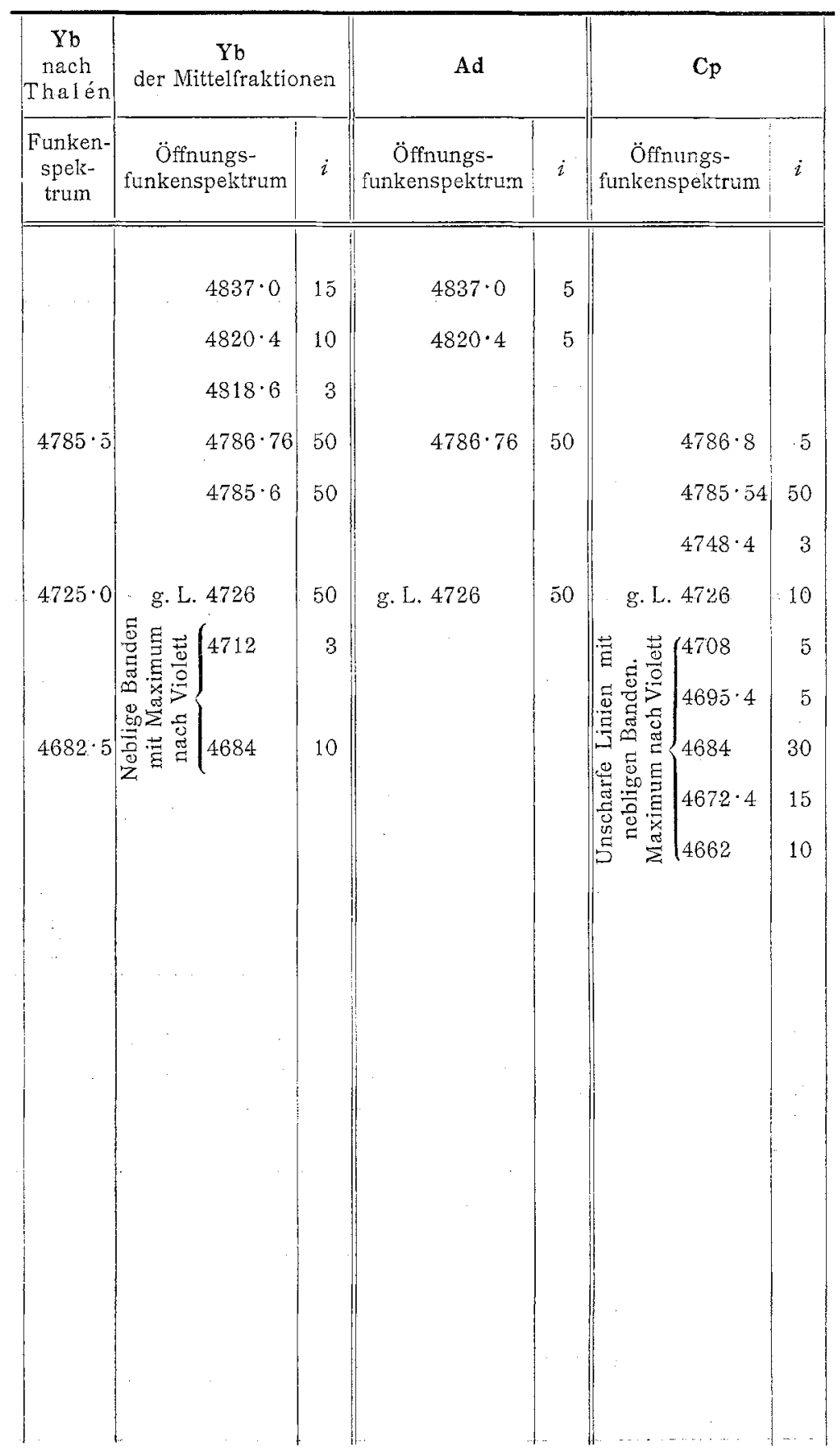




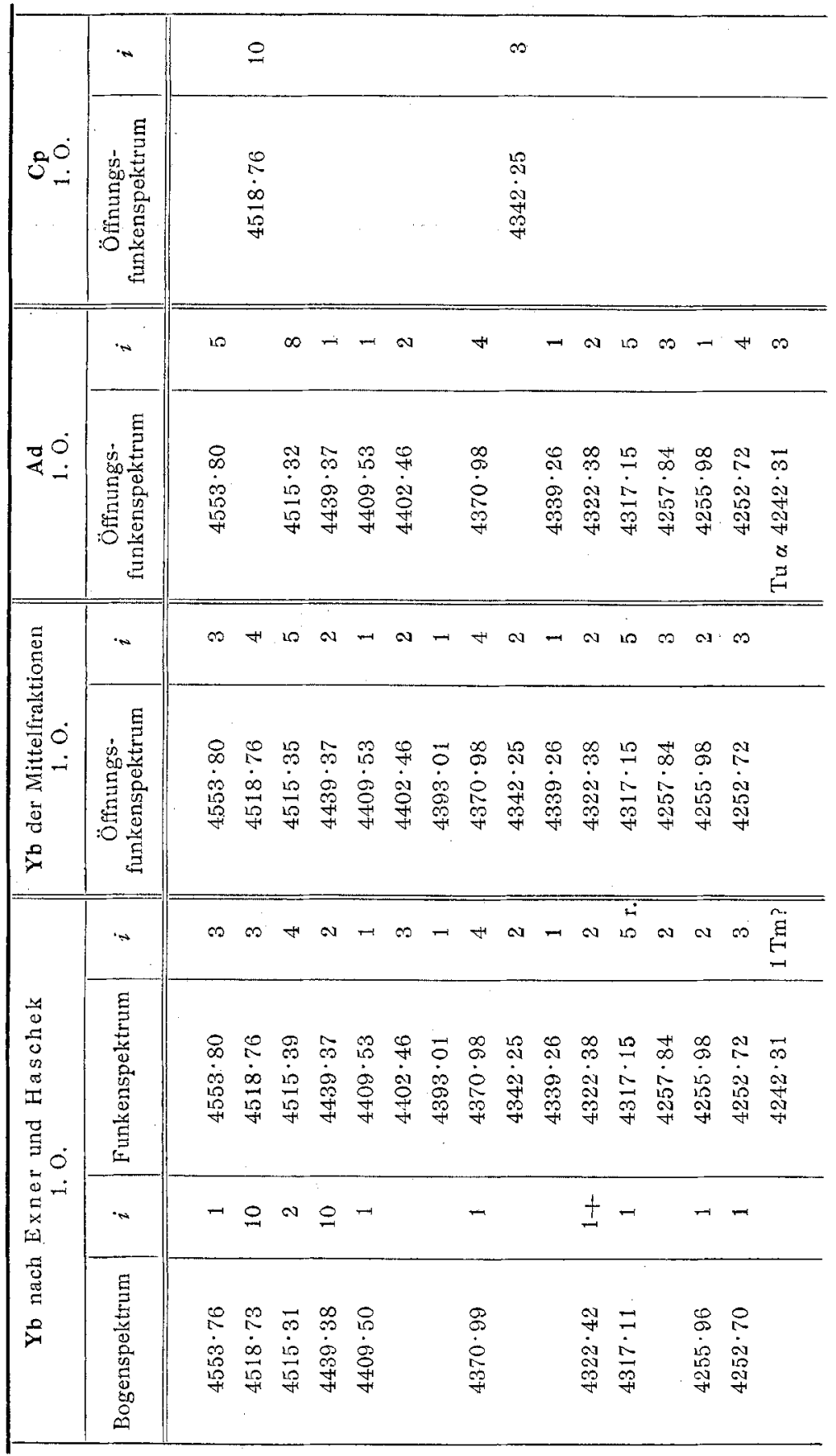




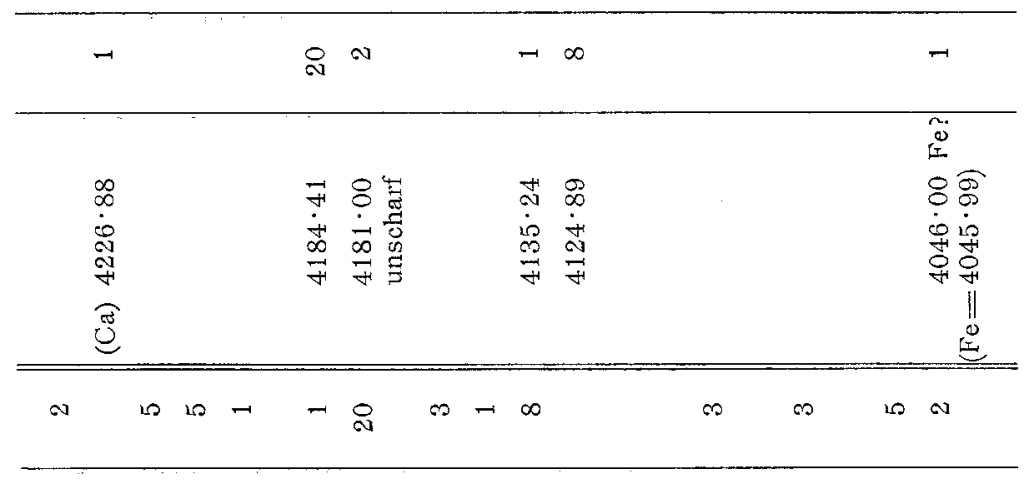

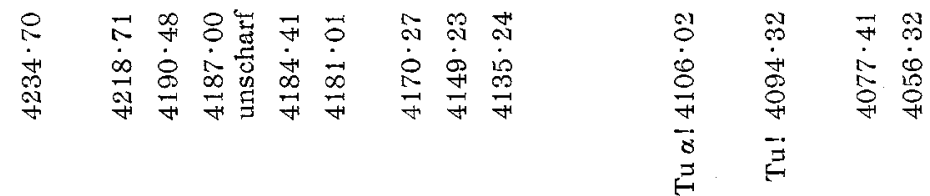

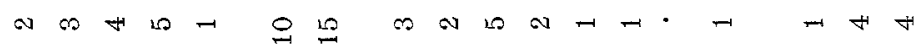

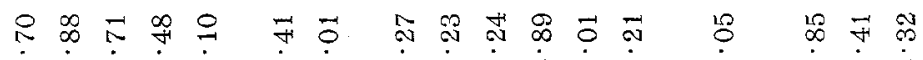

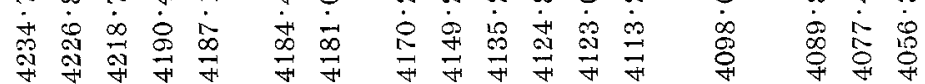

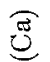

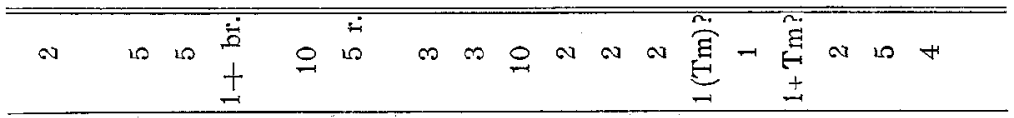

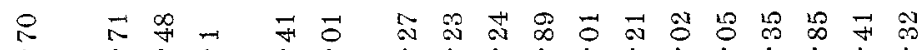

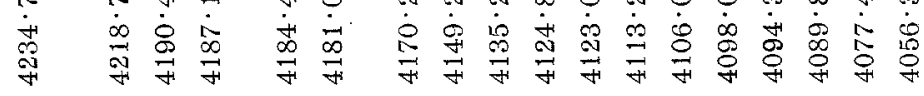

\begin{tabular}{|c|c|c|c|c|c|}
\hline+ \pm & +10 & $\rightarrow \infty \approx 10$ & $\widehat{E}$ & $\widehat{E}$ & $-\rightarrow$ \\
\hline
\end{tabular}

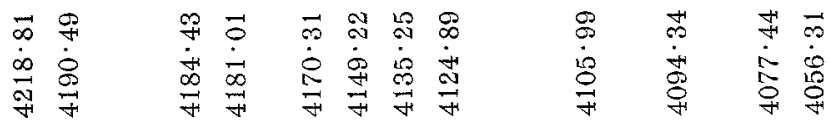




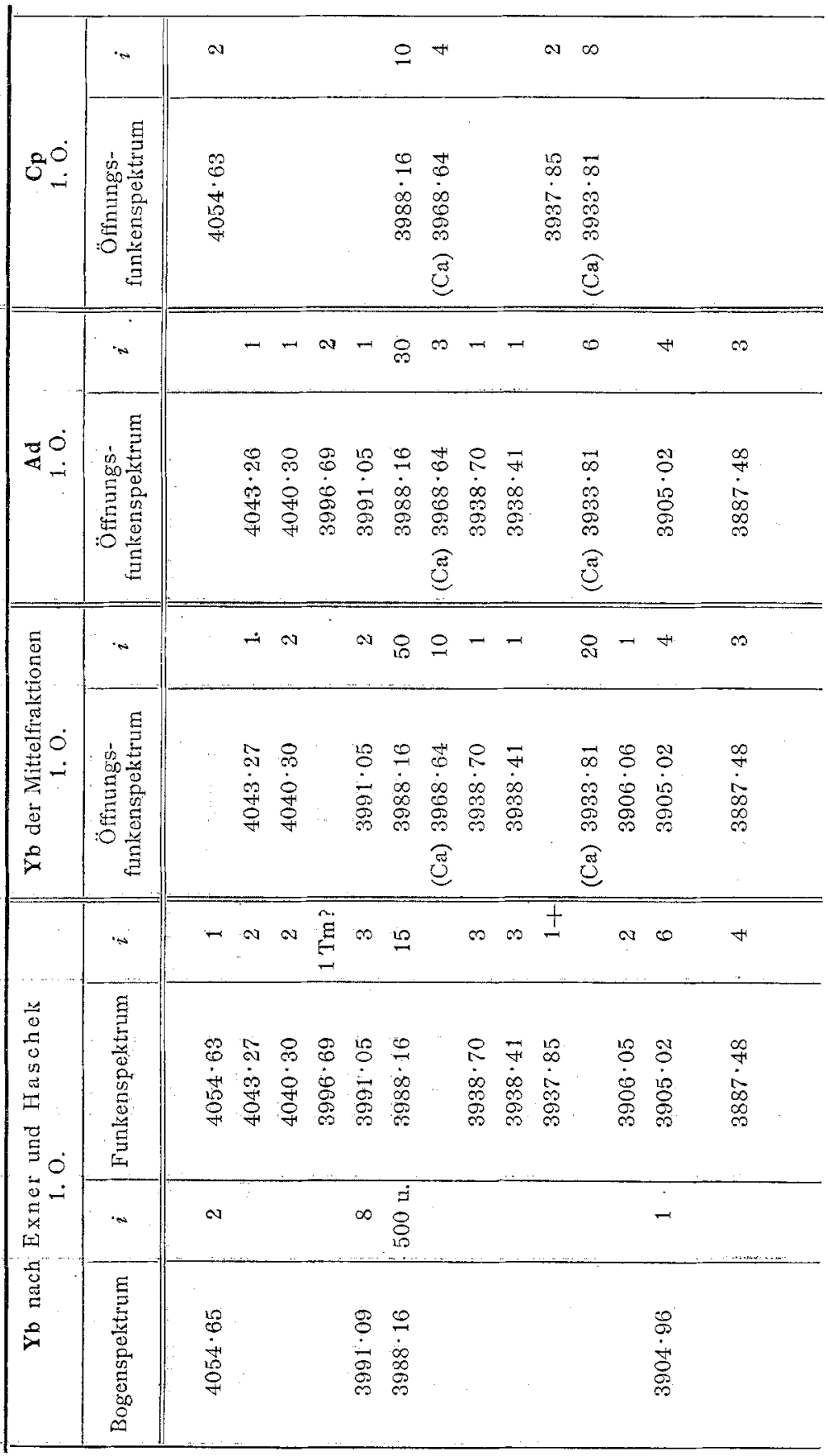




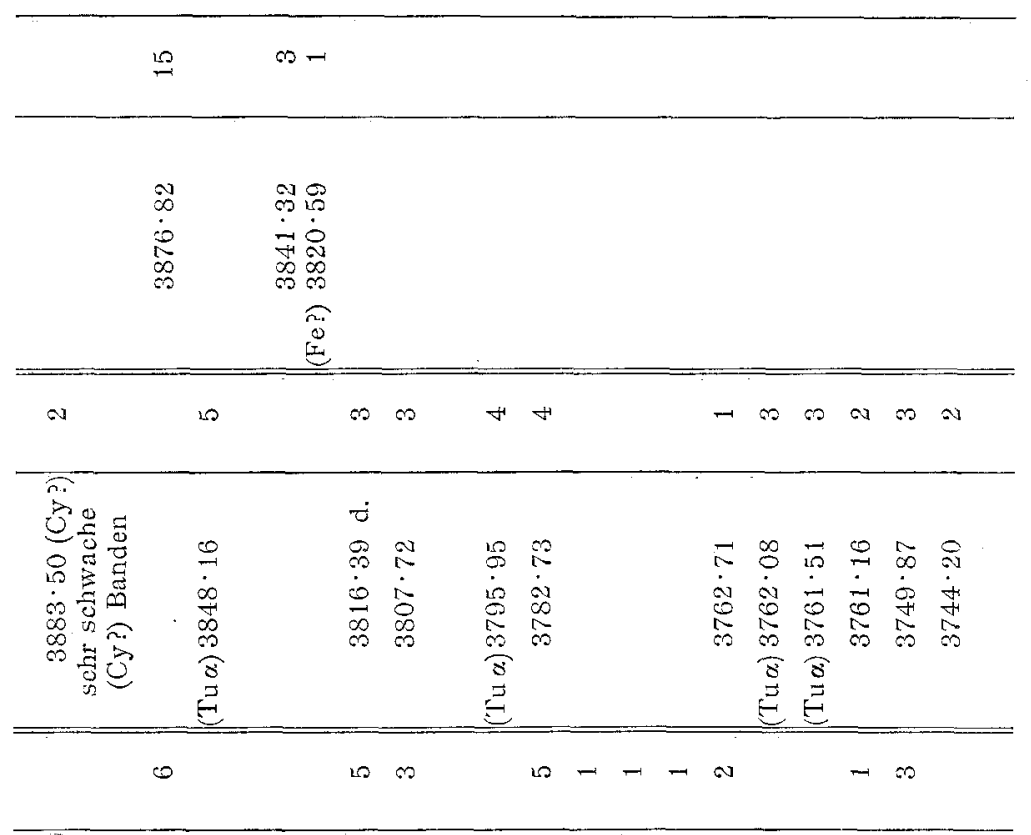

\begin{tabular}{|c|c|c|c|c|c|c|c|c|c|c|c|c|c|}
\hline 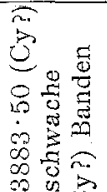 & $\underset{\infty}{\infty}$ & $\begin{array}{l}\dot{D} \\
\infty \\
\infty \\
\infty \\
\infty \\
\infty\end{array}$ & $\stackrel{0}{0}$ & $\underset{\infty}{\infty}$ & $\begin{array}{l}\infty \\
0 \\
0\end{array}$ & $\begin{array}{l}\overrightarrow{6} \\
\stackrel{8}{0}\end{array}$ & $\begin{array}{l}+1 \\
0 \\
0 \\
0 \\
0 \\
\infty\end{array}$ & $\begin{array}{l}10 \\
0 \\
0 \\
0\end{array}$ & & & $\frac{0}{\infty}$ & $\begin{array}{l}\infty \\
\dot{\infty} \\
\dot{\sigma} \\
\dot{\infty}\end{array}$ & \\
\hline & \pm & 0 & $\dot{\nabla}$ & 10 & $\infty$ & $\infty$ & $\infty$ & $\infty$ & 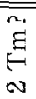 & 栗 & $\infty$ & $\dot{\nabla}$ & \pm \\
\hline
\end{tabular}

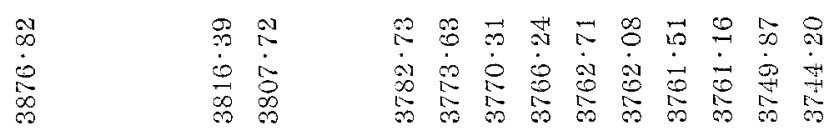

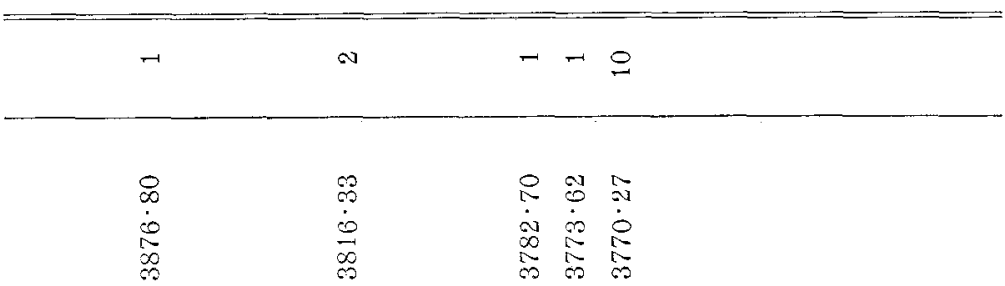


C. Auer v. Welsbach,

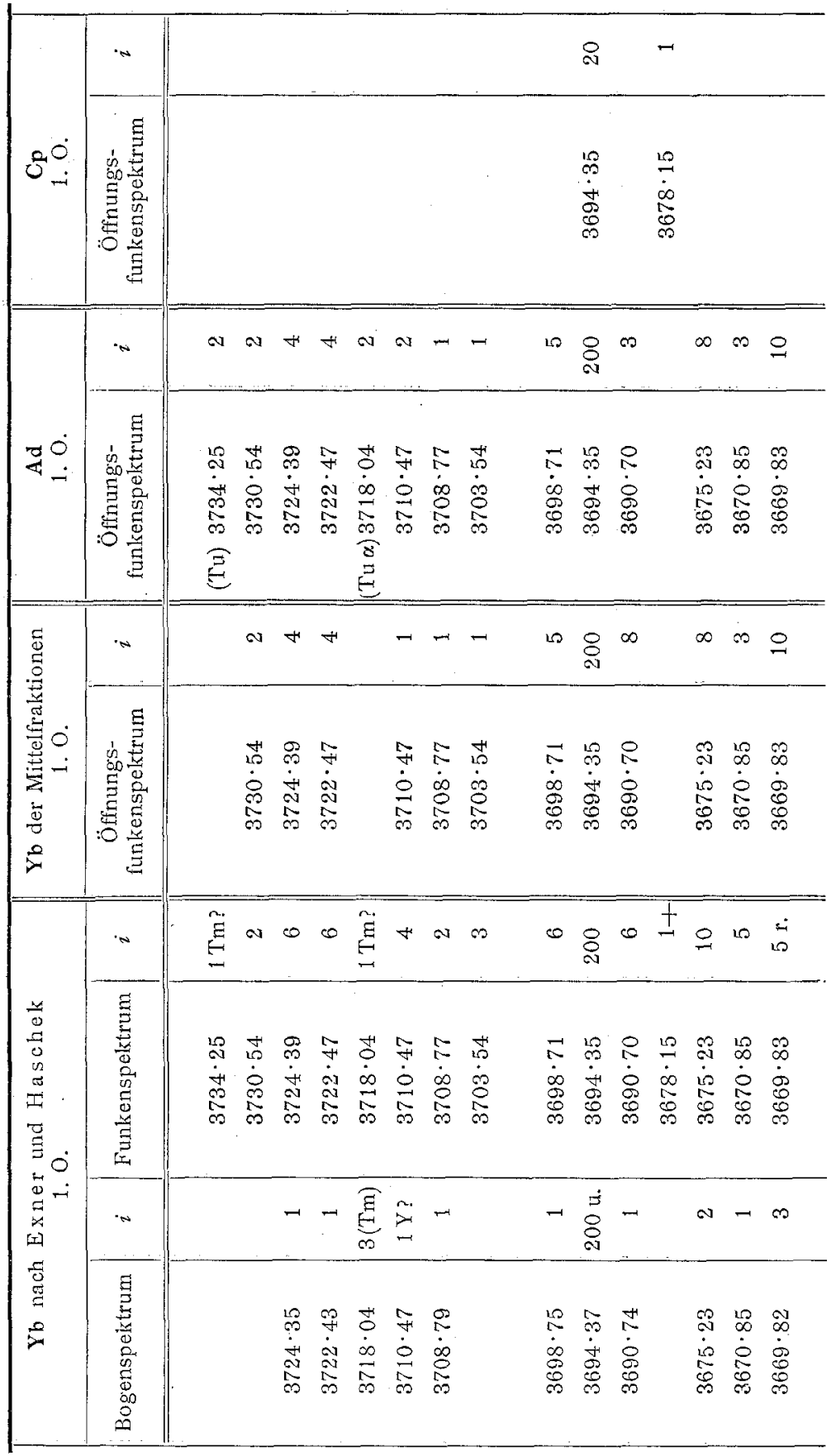




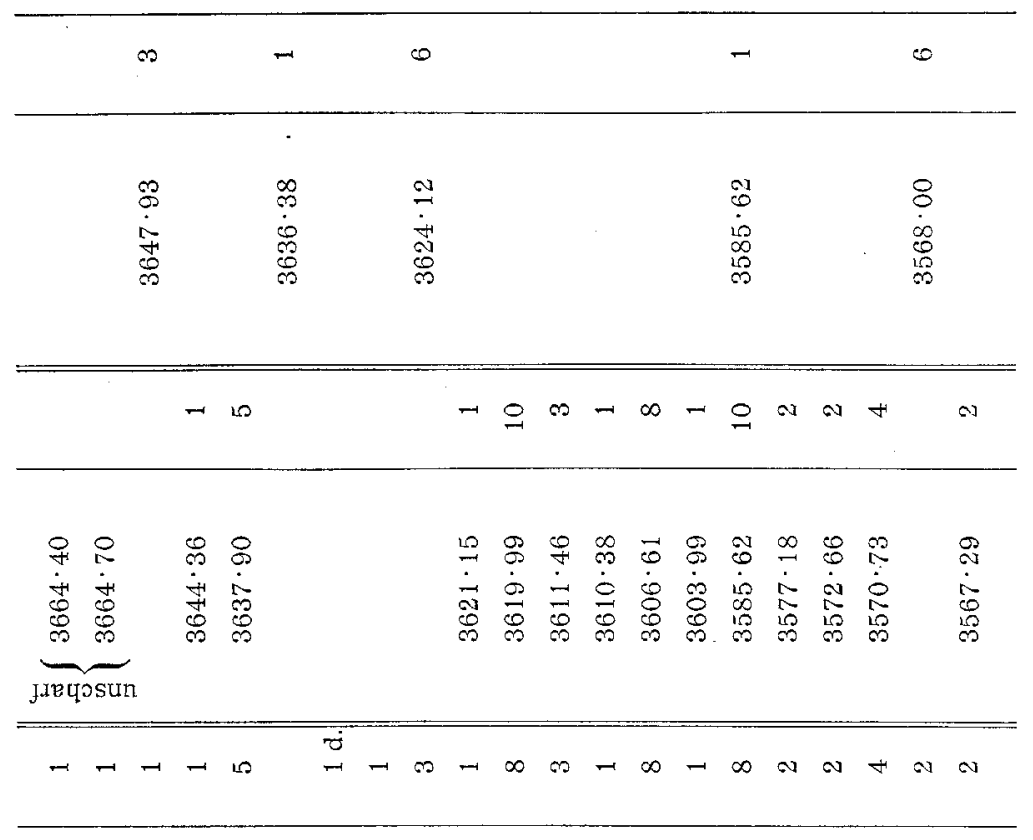

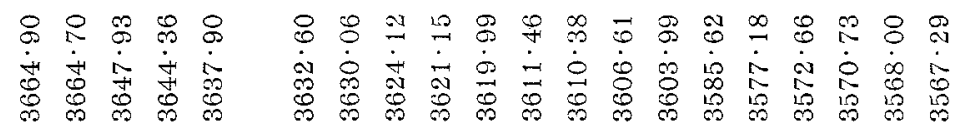

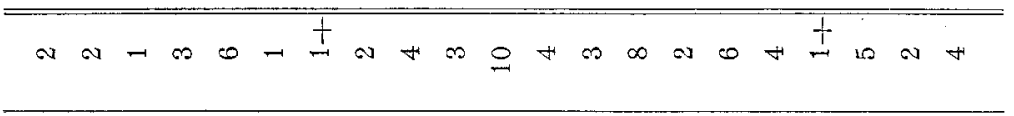

요

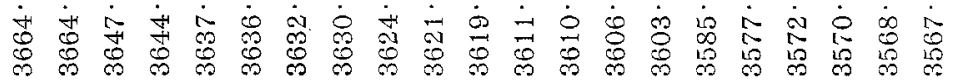

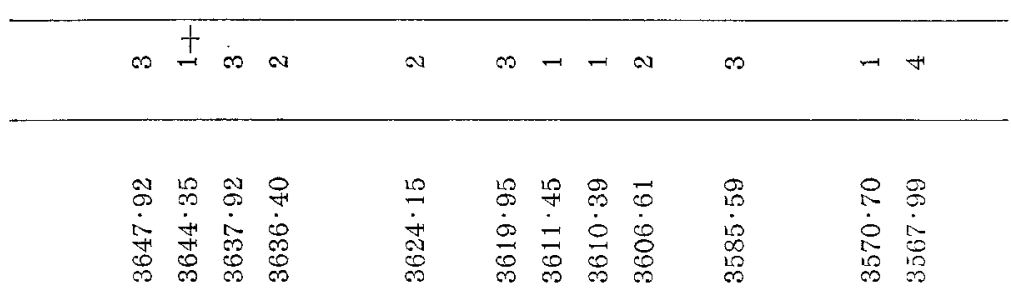




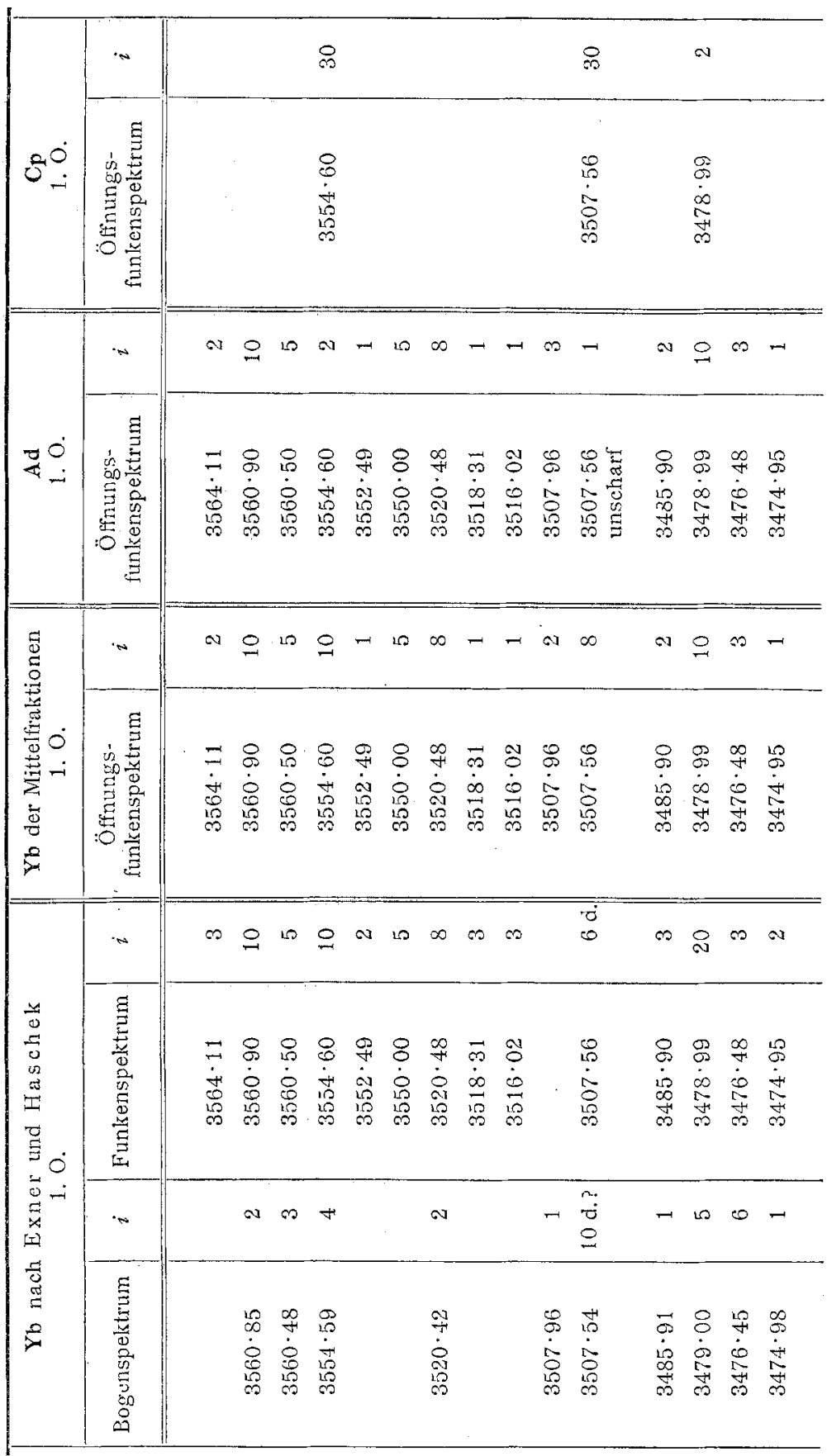




\begin{tabular}{|c|c|c|}
\hline 8 & -1 & 0 \\
\hline 9 & is & 10 \\
\hline$\dot{0}$ & + & $\ddot{n}$ \\
\hline I & 约尔 & $\sigma$ \\
\hline$\vec{\infty}$ & 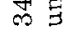 & $\infty$ \\
\hline
\end{tabular}
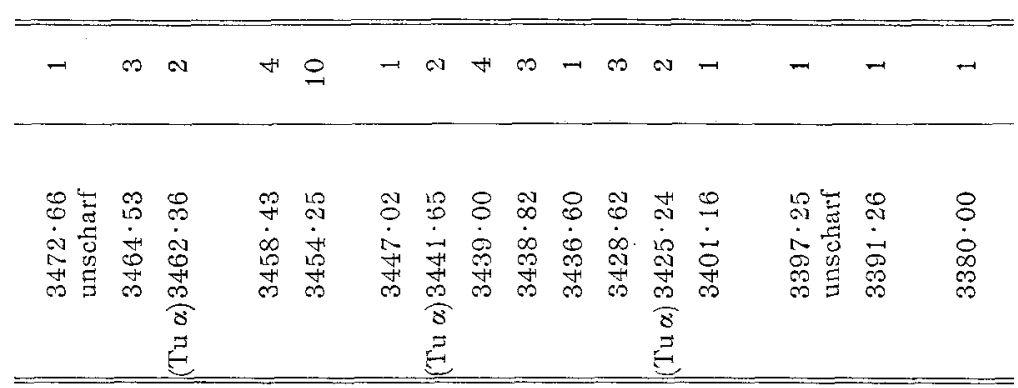

\begin{tabular}{|c|c|c|c|c|c|c|c|c|c|c|c|c|c|}
\hline 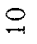 & $\infty$ & $\neg$ & 10 & 9 & $\rightarrow$ & षं & $\infty$ & $\rightarrow$ & $\infty$ & - & $\varrho$ & -1 & $\rightarrow$ \\
\hline
\end{tabular}

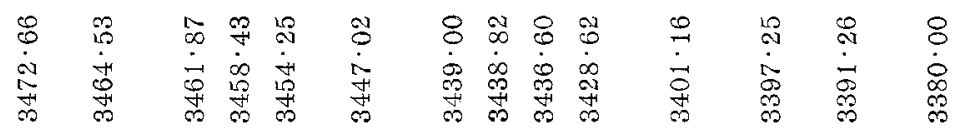

\begin{tabular}{|c|c|c|c|c|c|c|c|c|c|c|c|c|c|c|c|c|c|}
\hline 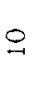 & $\infty$ & $\vec{E}$ & $\infty$ & $L \rho$ & $\stackrel{10}{\longrightarrow}$ & $\infty$ & $\sum_{-1}$ & 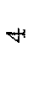 & $\infty$ & $\infty$ & $\nabla^{\prime}$ & $\tilde{E}$ & $\infty$ & $\varrho$ & $\infty$ & -1 & 60 \\
\hline
\end{tabular}

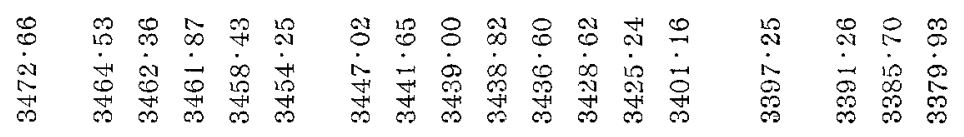

\begin{tabular}{|c|c|c|c|c|c|c|c|c|c|c|c|c|c|}
\hline 0 & $\begin{array}{l}\dot{1} \\
+ \\
0\end{array}$ & - & $\infty$ & $\begin{array}{l}\dot{4} \\
\infty\end{array}$ & -1 & $\infty u$ & - & $\rightarrow$ & - & - & 10 & $\neg \approx$ & -1 \\
\hline
\end{tabular}

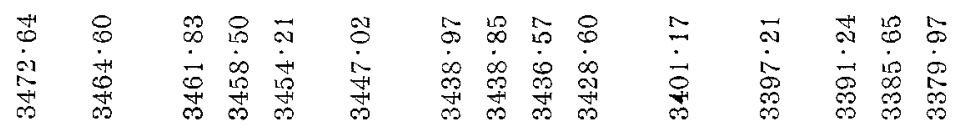




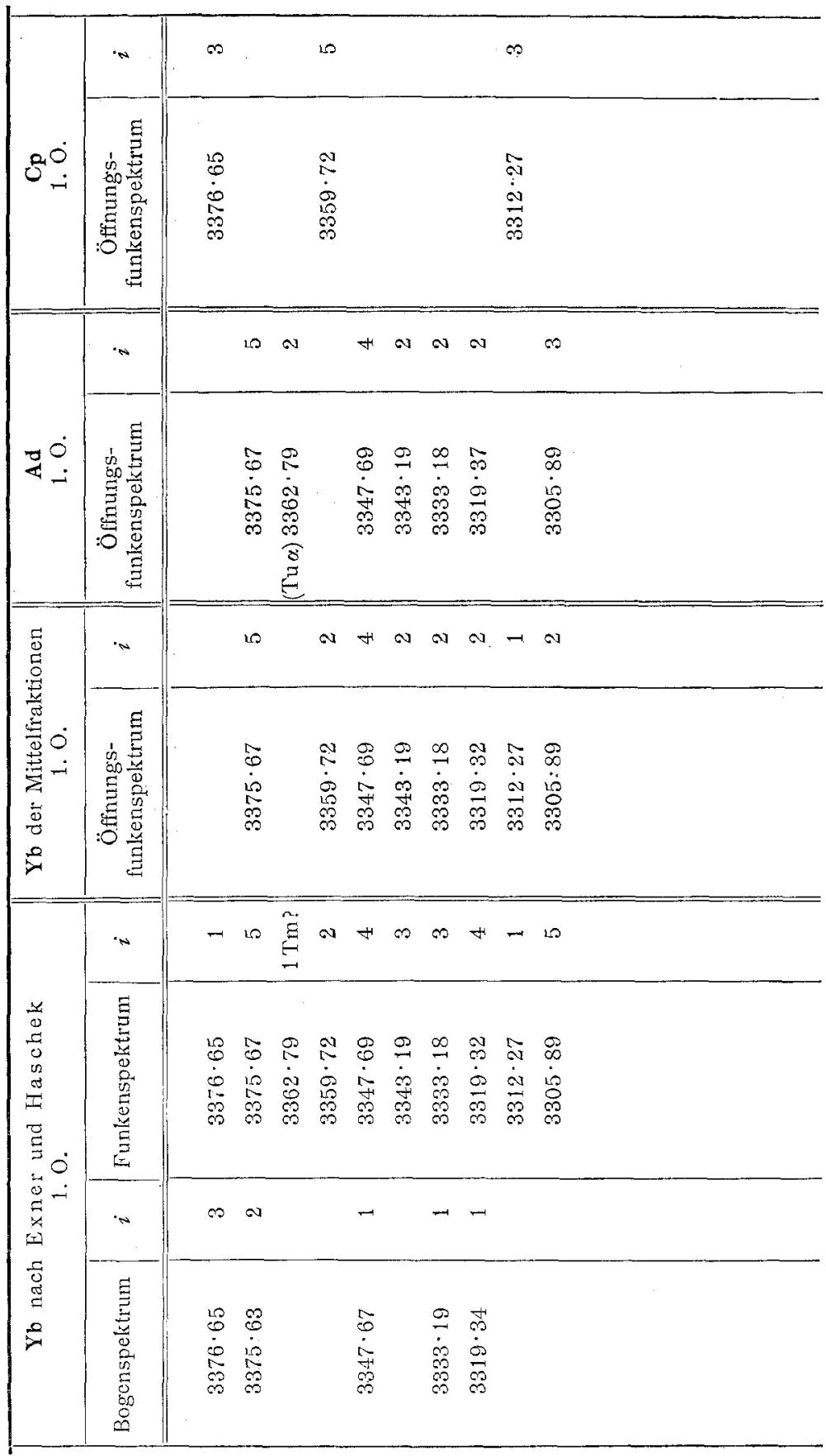


Zerlegung des Ytterbiums.

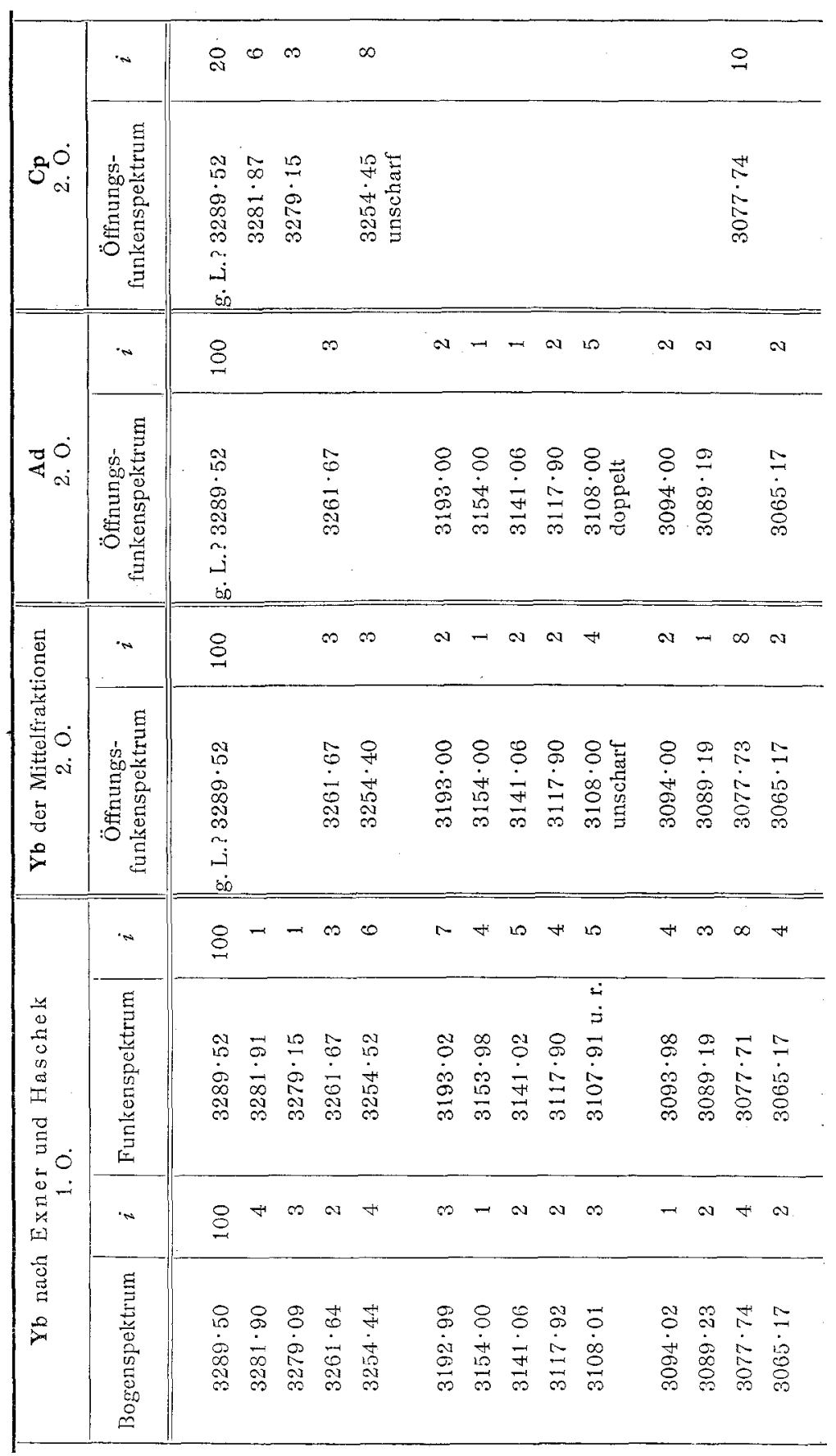




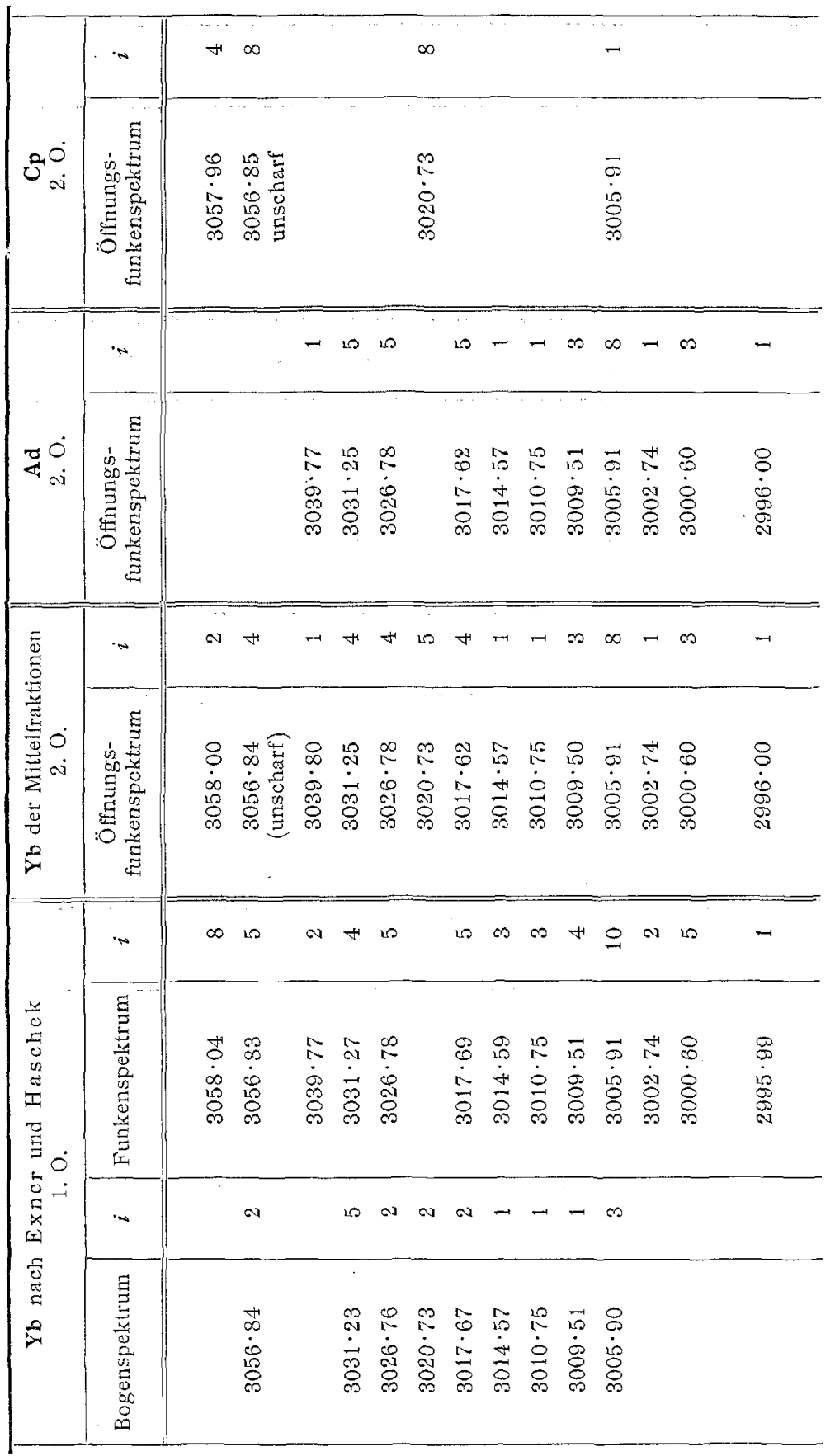


Zerlegung des Ytterbiums.

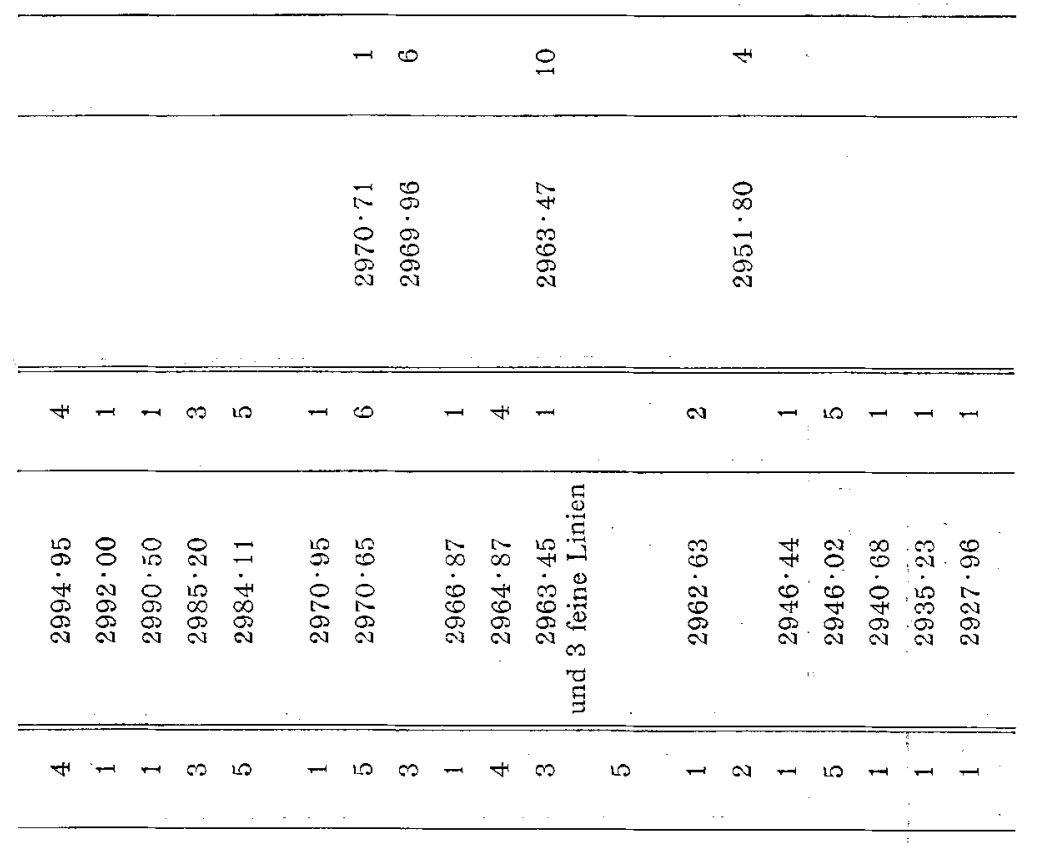

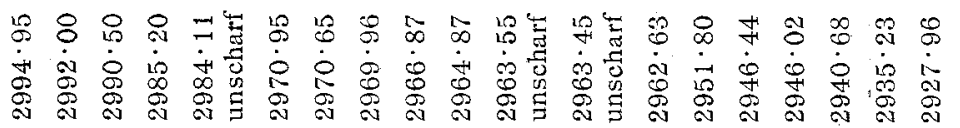

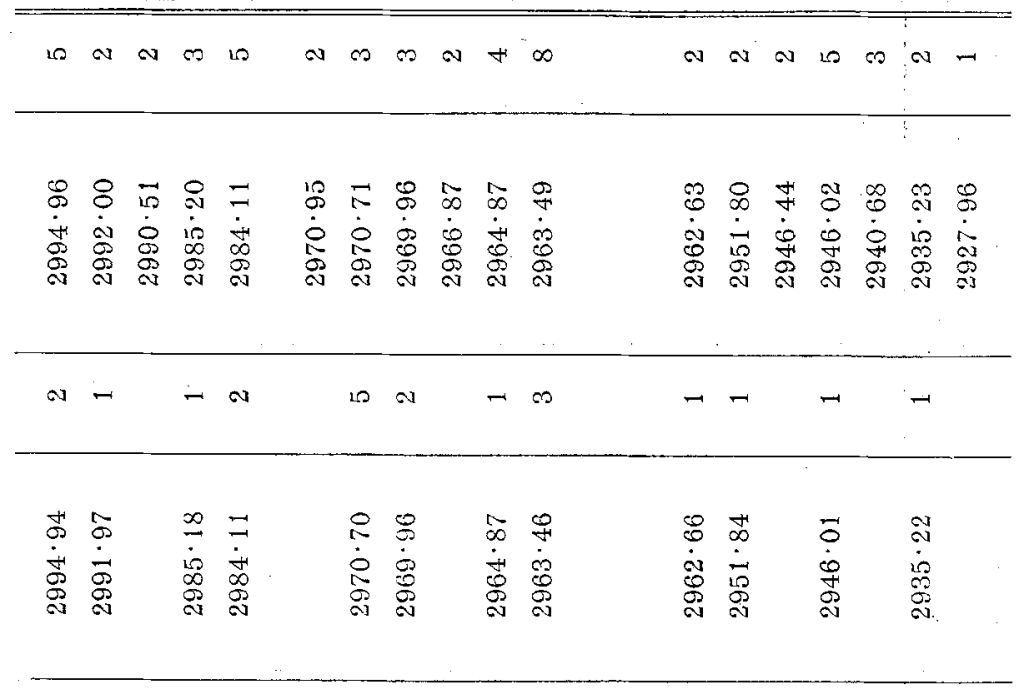


C. Auer v. Welsbach,

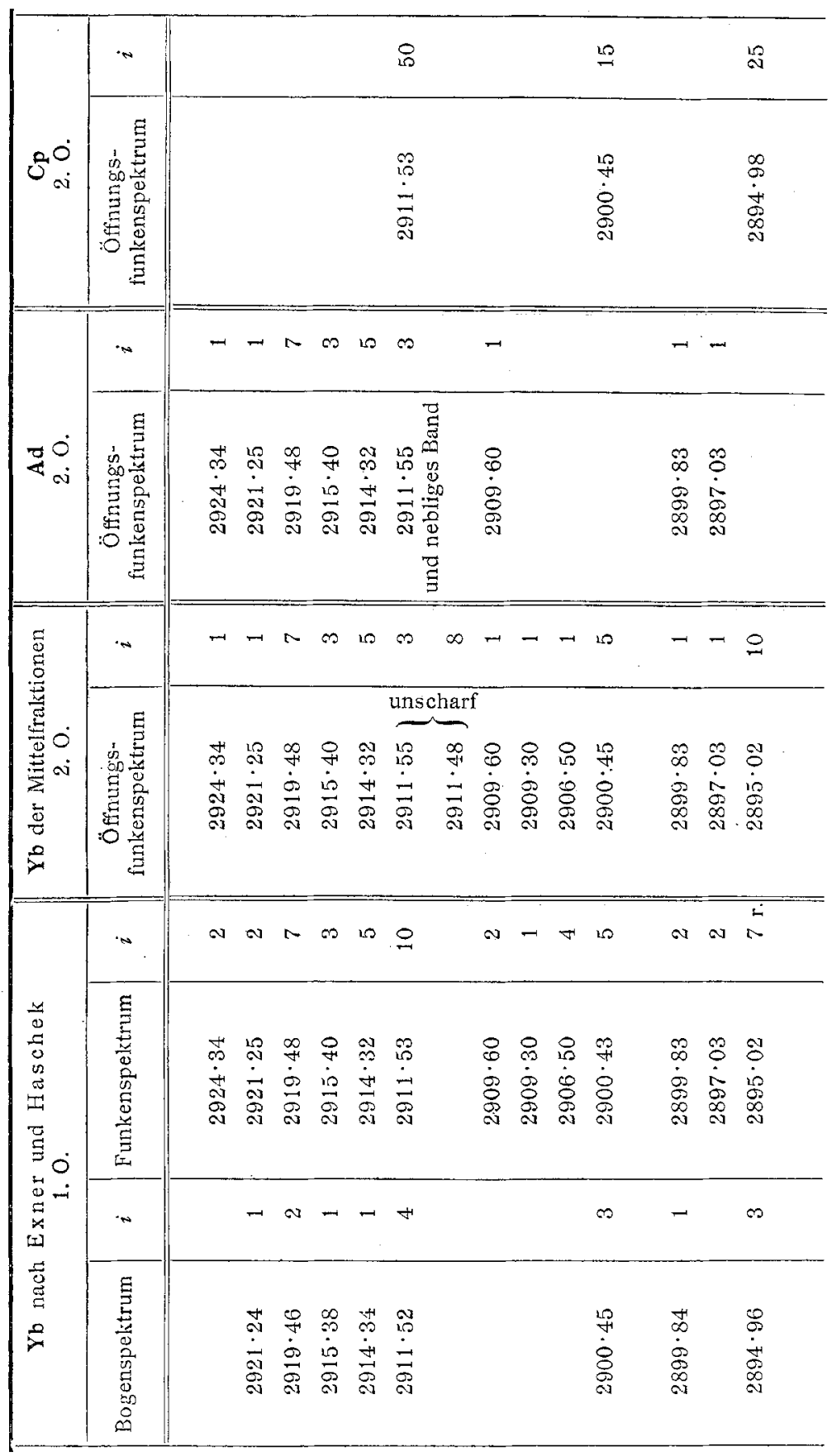



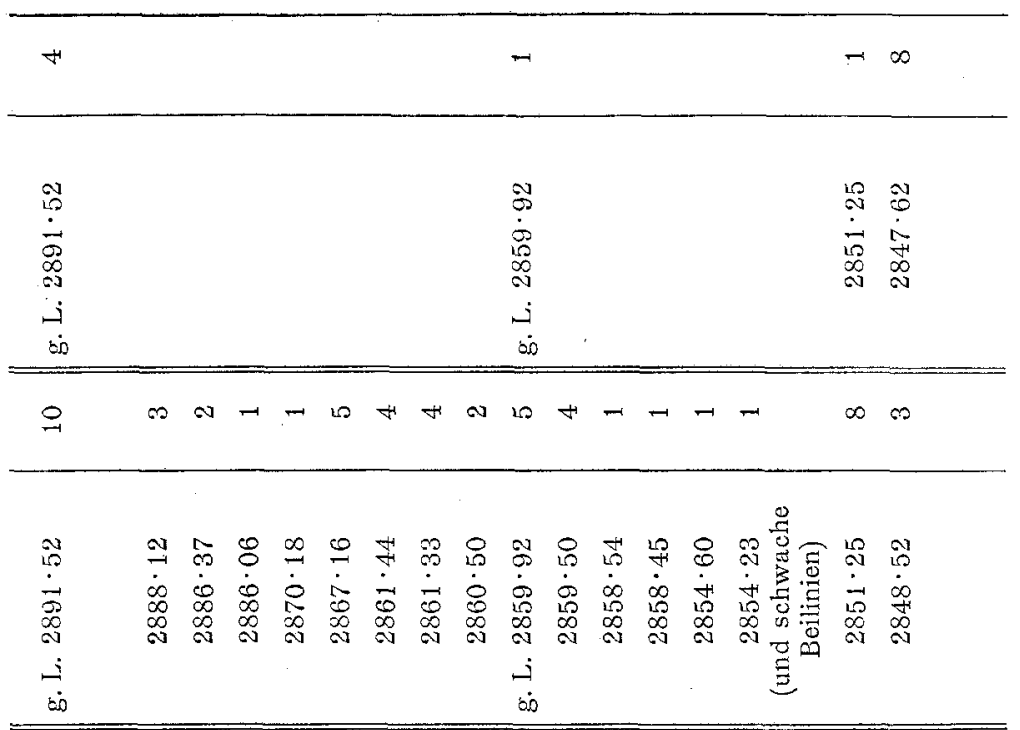

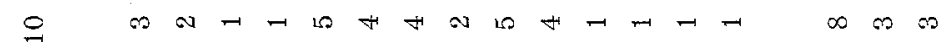

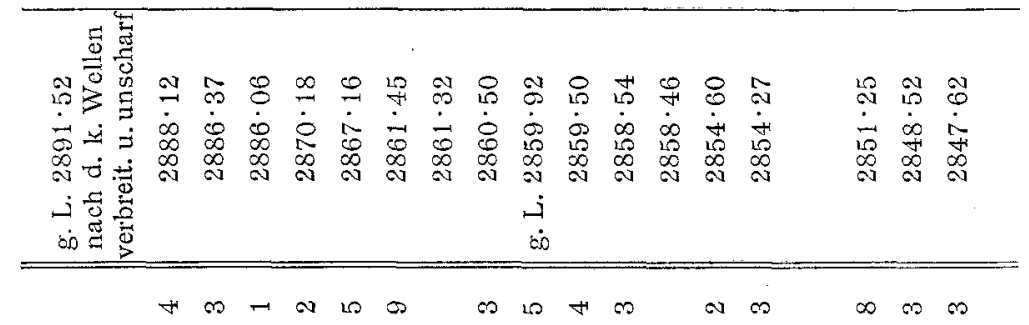

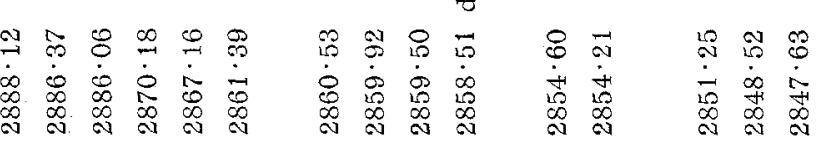

$\infty \rightarrow-\infty+-\infty+-\infty+\infty)$

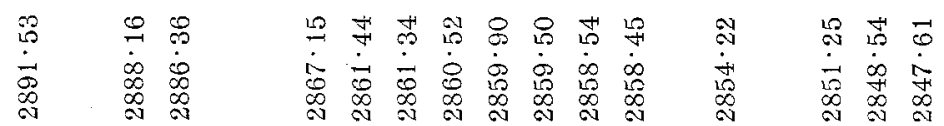




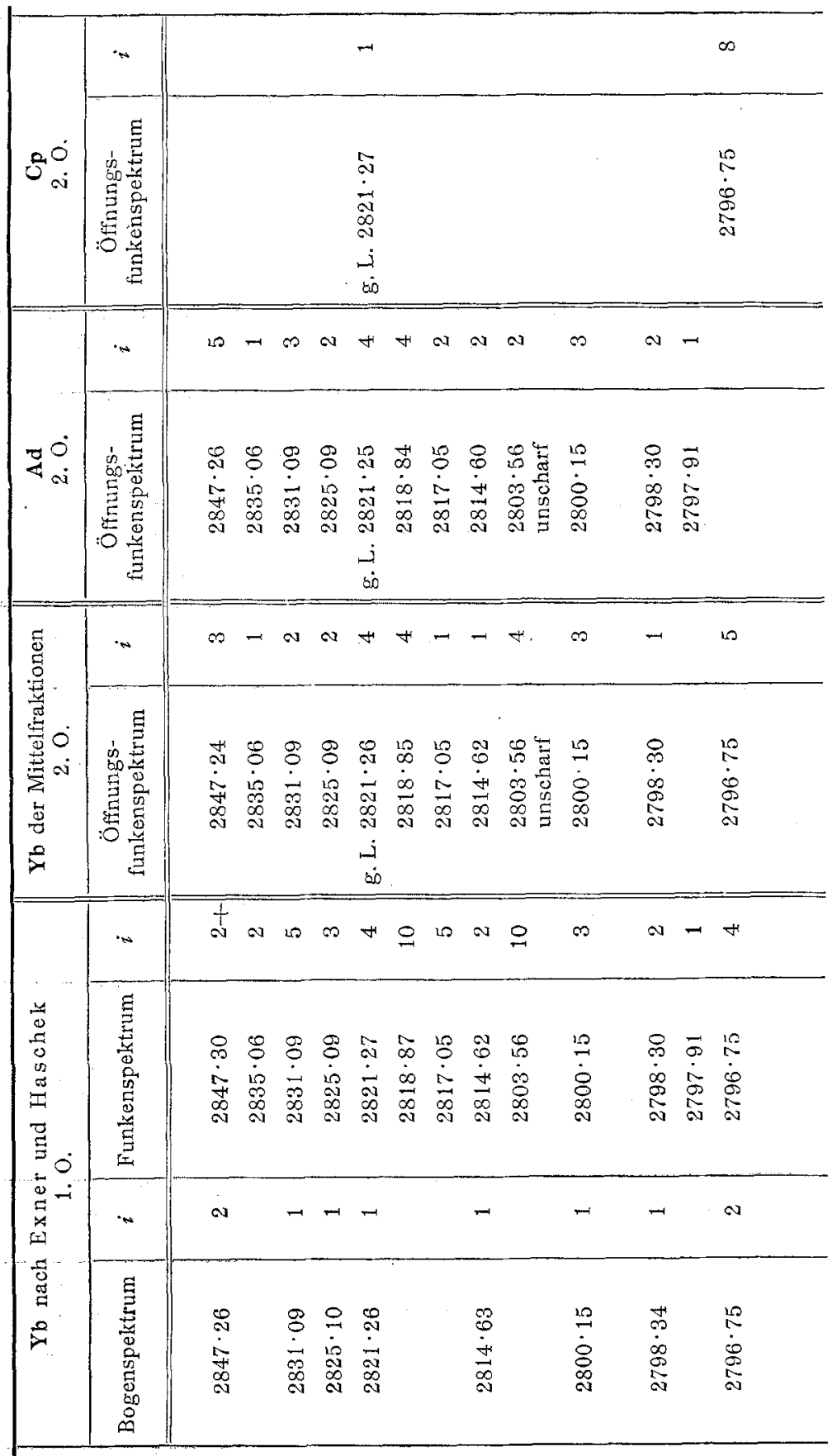


Zerlegung des Ytterbiums.
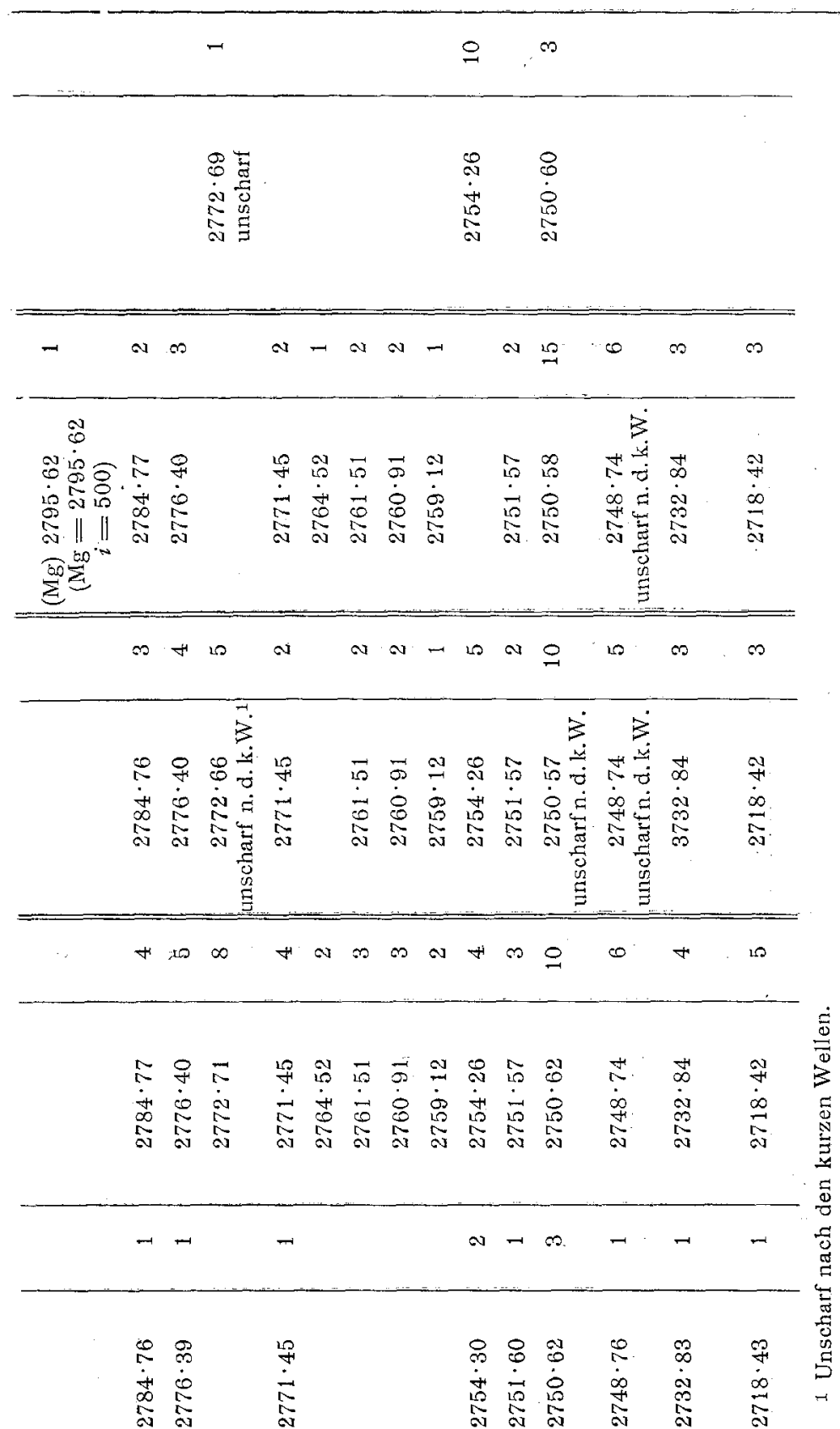


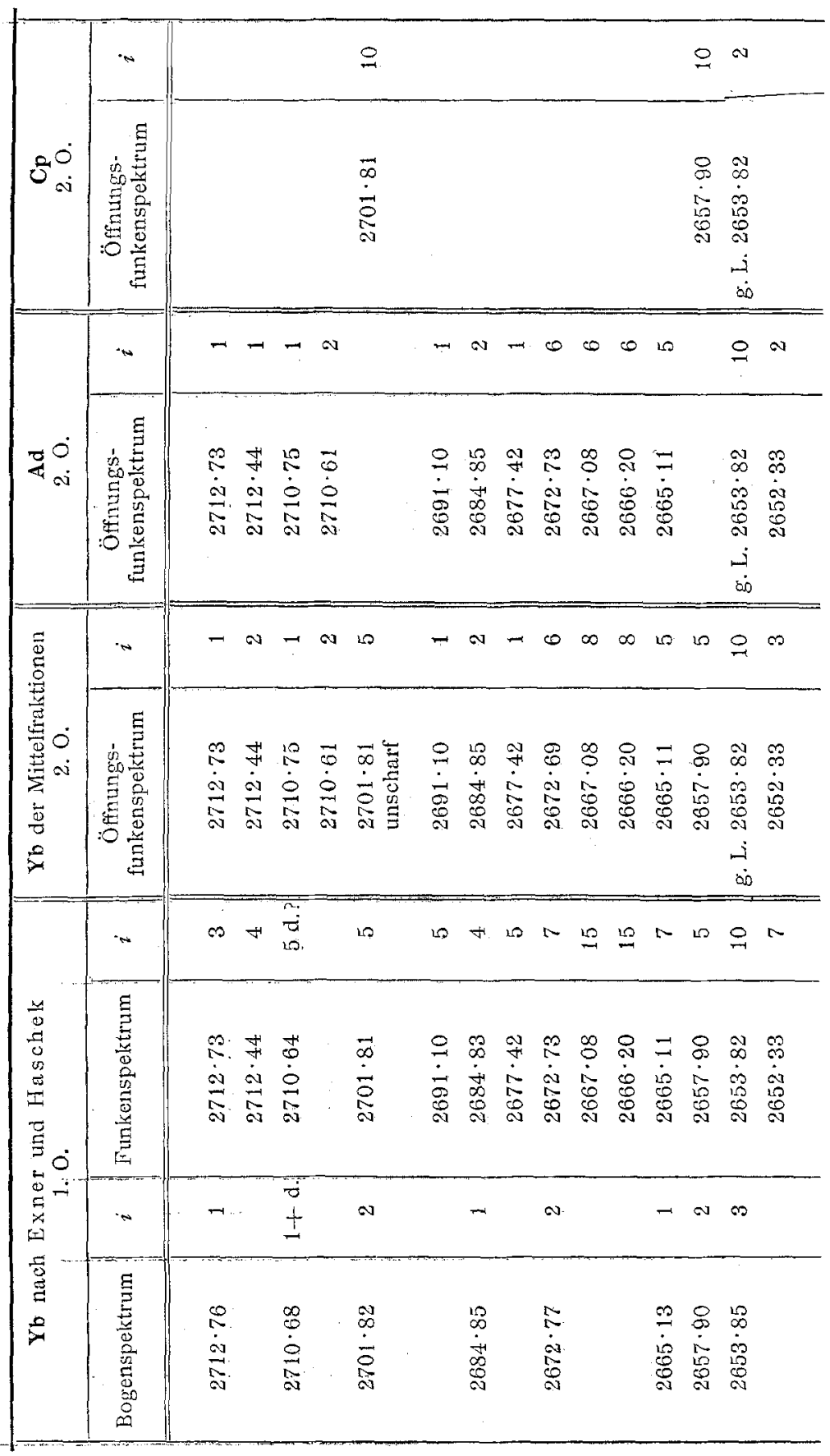


Zeriegung des Ytterbiums.

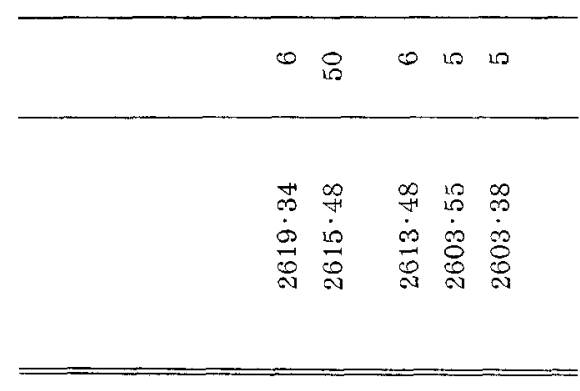

$\therefore a 0-100$

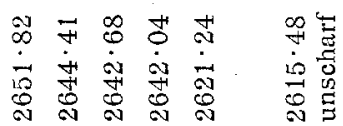

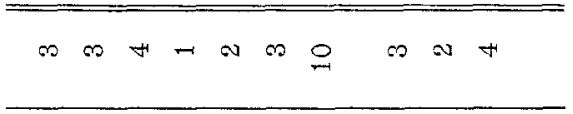

क Ұ

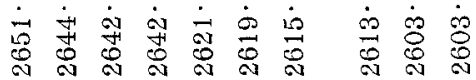

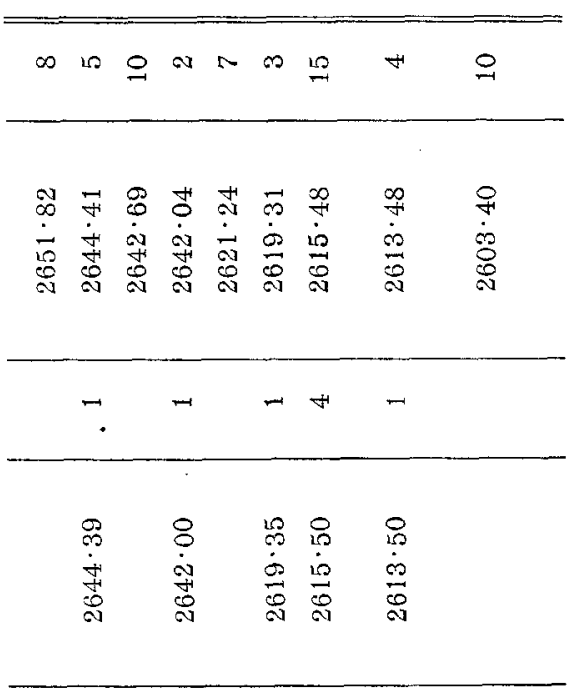

